\title{
Paleoclassical transport in low-collisionality toroidal plasmas
}

\author{
J. D. Callen ${ }^{\text {a) }}$ \\ University of Wisconsin, Madison, Wisconsin 53706-1609
}

(Received 1 July 2005; accepted 9 August 2005; published online 26 September 2005)

\begin{abstract}
Radial electron heat transport in a low-collisionality, current-carrying resistive plasma confined in an axisymmetric toroidal magnetic field is hypothesized to be caused by the paleoclassical collisional processes of parallel electron heat conduction and radial magnetic-field diffusion. The electron distribution is Maxwellianized and the electron temperature equilibrated over a long length $L$ ( $\gg$ the poloidal periodicity half-length $\pi R_{0} q$ ) along helical magnetic-field lines that are diffusing radially with the resistivity-induced magnetic-field diffusivity $D_{\eta} \equiv \eta_{\|}^{\text {nc }} / \mu_{0} \simeq \nu_{e}\left(c / \omega_{p}\right)^{2}$. This produces a paleoclassical radial electron heat diffusivity $\chi_{e}^{\mathrm{pc}}$ that is a multiple $M \simeq L /\left(\pi R_{0} q\right)$ $\sim 10 \gg 1$ of the magnetic-field diffusivity: $\chi_{e}^{\mathrm{pc}} \simeq(3 / 2) M D_{\eta}$. New paleoclassical model developments in this paper include full axisymmetric toroidal magnetic-field geometry, evolution of toroidal, poloidal, and helical magnetic fluxes, effects of temporally varying magnetic fluxes, introduction of electron guiding center radial diffusion effects induced by poloidal magnetic-flux diffusion into electron drift kinetics via a Fokker-Planck procedure, and determination of both axisymmetric and the usually dominant helically resonant paleoclassical radial electron heat transport. (c) 2005 American Institute of Physics. [DOI: 10.1063/1.2047227]
\end{abstract}

\section{INTRODUCTION}

A new "paleoclassical transport" paradigm has recently been hypothesized; ${ }^{1}$ it provides interpretations ${ }^{1-3}$ for most "anomalous" electron heat transport observed in lowcollisionality toroidal plasmas (for $T_{e} \leqslant 2 \mathrm{keV}$ ). The key new physical point is that as magnetic-field lines diffuse radially (with $D_{\eta} \simeq \eta_{0} / \mu_{0} \equiv \nu_{e} c^{2} / \omega_{p}^{2}$, which implies a diffusive process with $\Delta x \sim \delta_{e} \equiv c / \omega_{p}$ in a time $\left.\Delta t \sim 1 / \nu_{e}\right)$, they carry with them electron heat equilibrated over a long parallel length $L$, which is the minimum of the electron collision length $\lambda_{e}$ and a maximum effective field-line length. Because $L$ is much longer than the poloidal periodicity length $\pi R_{0} q$, the induced radial electron heat diffusivity is of order $M$ $\simeq L / \pi R_{0} q \sim 10 \gg 1$ times $D_{\eta}$. Since the initial paleoclassical model, which used a sheared slab magnetic-field model, required adaptations to represent toroidal geometry effects, this paper develops the model further by calculating the paleoclassical radial electron heat transport in an axisymmetric toroidal geometry-to make it applicable to most types of toroidal plasma experiments.

In a 1970 paper $^{4}$ Grad and Hogan made a prophetic statement: "plasma diffusion is a complex phenomenon with at least two distinct diffusive time scales nonlinearly coupling field diffusion (the skin effect), plasma diffusion, plasma convection, and geometrical effects...." The magnetic-field diffusion is the faster time-scale process since the magnetic-field diffusivity $D_{\eta}$ is larger than the classical or neoclassical diffusivity—because the electromagnetic skin depth $\delta_{e}$ is usually larger than the electron gyroradius or banana width. Thus, magnetic-field lines diffuse radially faster than collisions cause electrons to diffuse relative to them. The Grad and Hogan paper and subsequent papers by

\footnotetext{
a)Electronic mail: callen@engr.wisc.edu; URL: http://www.cae.wisc.edu/ callen
}

Pao, ${ }^{5,6}$ as well as the standard review papers ${ }^{7,8}$ for neoclassical Pfirsch-Schlüter and banana-plateau transport, determined axisymmetric plasma transport in the presence of magnetic-field diffusion. However, in these and subsequent papers the field diffusion effects were only considered within the context of plasma fluid models; in particular, the magnetic-field diffusion did not influence the kinetic analysis.

The key hypothesis of the paleoclassical model is to add effects of radial magnetic-flux (field line) diffusion to electron drift-kinetic theory-by including in the drift-kinetic equation a spatial Fokker-Planck operator that represents the effects of magnetic-field diffusion on electron guiding center motion. Such a Fokker-Planck treatment is appropriate because the poloidal magnetic flux traversed by an electron's gyromotion diffuses radially due to magnetic-field diffusion. This causes the radial position of an electron's guiding center in current-carrying resistive toroidal plasmas such as tokamaks in which the poloidal magnetic field is a drivendissipative (i.e., nonconservative) field to not be a "collisionless" constant of motion, as is usually assumed, but to become a "stochastic" (diffusing) variable—see Sec. VI.

The initial paleoclassical model ${ }^{1}$ used a sheared slab magnetic-field geometry and assumed, for simplicity, the magnetic field was stationary. This paper develops the paleoclassical model for a general axisymmetric toroidal magnetic-field geometry, and allows for temporally evolving magnetic fluxes and field lines. Also, the full Fokker-Planck procedure for including effects of the radial advection and diffusion of field lines on electron guiding centers in electron drift-kinetic theory is developed. Further, the kinetics of the Maxwellianization of the electron distribution along axisymmetric (poloidal) and nonaxisymmetric (helical) field lines that are diffusing radially is developed. Finally, a ballooningtype representation for the distribution function is developed 
and used to preserve poloidal and helical symmetries in the paleoclassical "flute-like" transport responses in the vicinity of medium-order rational surfaces defined by $q_{*} \equiv m / n$ with $n \leqslant n_{\max } \simeq 1 /\left(\pi \delta_{e} d q / d r\right)^{1 / 2} \sim 10$-see (121).

This paper is organized as follows. Section II discusses key properties of the axisymmetric magnetic-field model used throughout the paper. Section III uses components of Faraday's law to develop evolution equations for the toroidal and poloidal magnetic fluxes in terms of appropriate components of the electric field. Section IV develops a relevant (flux-surface-averaged, neoclassical plus inertia) parallel Ohm's law. The motion and diffusion of magnetic-flux surfaces and field lines, and, in particular, helical fluxes in the vicinity of rational surfaces, are explored in Sec. V. The effects of magnetic-field diffusion on electron guiding center motion and the resultant drift-kinetic equation for paleoclassical processes, which includes a Fokker-Planck spatial operator representing the effects induced by the radial diffusion of poloidal magnetic flux, is developed and solved in Sec. VI. Thereafter, in Sec. VII an electron energy balance equation for paleoclassical processes is obtained and its consequences explored. Section VIII summarizes the main contributions of this paper.

\section{AXISYMMETRIC MAGNETIC-FIELD GEOMETRY}

The initial paper $^{1}$ on paleoclassical radial electron heat diffusion used a sheared slab magnetic-field model. Here, to more accurately describe relevant magnetic-field geometries and to clarify some ambiguities that arise in the sheared slab model, a full axisymmetric magnetic-field model is used. In what follows the paleoclassical model is developed for arbitrary aspect ratio $\left(A \equiv R_{0} / r \equiv 1 / \epsilon\right.$ where $R_{0}, r$ are the major, minor radii of a flux surface) to facilitate application of the paleoclassical model to most types of axisymmetric toroidal plasmas-large aspect ratio tokamaks $(A \gg 1)$, and regions of spherical tokamaks (STs, $A \gtrsim 1$ ), spheromaks, and reversed field pinches (RFPs) where the squares of the local inverse aspect ratio and ratio of the poloidal magnetic field $\left(B_{p}\right)$ to the toroidal magnetic field $\left(B_{t}\right)$ are small: $\epsilon^{2}, B_{p}^{2} / B_{t}^{2} \ll 1$. However, the theory is developed from a tokamak perspective in that normal arbitrary aspect ratio tokamak variables are used throughout. Approximate forms for large aspect ratio, nearly circular cross-section tokamaks are indicated at the end of many equations after an approximate equality $(\simeq)$.

The paleoclassical transport model is concerned with diffusion of magnetic flux. The first question that arises is diffusion relative to what coordinates? Hirshman and Jardin 9 have shown that for axisymmetric toroidal plasmas with $\epsilon^{2}, B_{p}^{2} / B_{t}^{2} \ll 1$ the toroidal magnetic flux $\psi_{t}(\mathbf{x}, t)$ is less mobile than the poloidal magnetic flux $\psi(\mathbf{x}, t)$-because $\psi$ is caused by self-consistent parallel ( $\sim$ toroidal) currents flowing in the plasma while $\psi_{t}$ is due predominantly to currents flowing poloidally in external coils. (For an approach that allows both poloidal and toroidal fluxes to be evolving see Strand and Houlberg. ${ }^{10}$ ) Transport fluxes are calculated ${ }^{7-9}$ relative to the (usually small) "radial grid velocity" of toroidal flux surfaces induced by the poloidal electric field. Thus, diffusion of the poloidal flux surfaces (and field lines) will be determined relative to the toroidal flux surfaces:

$$
\psi_{t}(\rho, t) \equiv \frac{1}{2 \pi} \iint d \mathbf{S}(\zeta) \cdot \mathbf{B}_{t} \simeq \frac{r^{2} B_{0}}{2}, \quad \text { toroidal flux },
$$

in which $B_{0}$ is the magnetic-field strength at the magnetic axis $\left(\psi_{t}=0\right)$ and the surface integral is over the crosssectional area of a given magnetic-flux surface in a constant toroidal angle plane. A convenient, dimensionless, cylindrical-type radial variable is $\rho$ :

$$
\rho \equiv \sqrt{\psi_{t} / \psi_{t}(a)} \simeq r / a, \quad \text { radial coordinate },
$$

in which $\psi_{t}(a)$ is the toroidal magnetic flux enclosed by the last closed flux surface in the plasma, which is of minor radius $a$ for a circular cross-section plasma.

The appropriate magnetic-field model ${ }^{8,9}$ for an axisymmetric toroidal plasma can be described in terms of its toroidal $(t)$ and poloidal $(p)$ components by

$$
\begin{aligned}
\mathbf{B}= & \mathbf{B}_{t}+\mathbf{B}_{p}=B_{t} \hat{\mathbf{e}}_{\zeta}+B_{p} \hat{\mathbf{e}}_{\theta}=I \boldsymbol{\nabla} \zeta+\boldsymbol{\nabla} \zeta \times \nabla \psi=\nabla \psi \\
& \times \boldsymbol{\nabla}(q \theta-\zeta)=\boldsymbol{\nabla} \times \mathbf{A}, \quad \text { for } \mathbf{A} \equiv \psi_{t} \boldsymbol{\nabla} \theta-\psi \nabla \zeta .
\end{aligned}
$$

Here, presuming an axisymmetric ideal magnetohydrodynamic (MHD) equilibrium (i.e., one that has an isotropic pressure to lowest order and whose magnetic flux satisfies the Grad-Shafranov equation-see, for example, Refs. 11 and 12) $I=I(\rho, t) \equiv R B_{t} \simeq B_{0} R_{0}$ is a current function that represents predominantly the currents flowing poloidally in the external coils in which $R \equiv 1 /|\nabla \zeta| \simeq R_{0}+\rho a \cos \theta+\mathcal{O}\left(\epsilon^{2} R_{0}\right)$ is the major radius to a given point on a flux surface. Also, $\zeta$ is the toroidal (axisymmetry, long way around the torus) angle and $\psi$ is the poloidal magnetic-flux function:

$$
\psi(\rho, t) \equiv \frac{1}{2 \pi} \iint d \mathbf{S}(\theta) \cdot \mathbf{B}_{p}, \quad \text { poloidal flux },
$$

for which $\partial \psi / \partial \rho \simeq a R_{0} B_{p}$. Further, $\theta$ is the poloidal (short way around torus) angle in which the magnetic-field lines are straight (in the $\psi=$ constant plane) and $q$ is the winding number or pitch ("safety factor" for kink stability) of magneticfield lines on a flux surface:

$$
q(\rho, t) \equiv \frac{\partial \psi_{t} / \partial \rho}{\partial \psi / \partial \rho}=\frac{\text { no. toroidal transits }}{\text { no. poloidal transits }} \simeq \frac{r B_{t}}{R_{0} B_{p}} .
$$

For an axisymmetric magnetic field $q(\rho, t)=q(\psi, t)$ and

$$
\text { B } \cdot \nabla \theta=I / q R^{2} \simeq B_{t} / R_{0} q=B_{p} / r .
$$

Some useful properties of this magnetic-field description and coordinate system are as follows. First, note that it is not an othogonal coordinate system since $\boldsymbol{\nabla} \rho \cdot \nabla \theta \propto \nabla \psi_{t} \cdot \nabla \theta \neq 0$. The Jacobian of the transformation from the original fixed Eulerian coordinates to the curvilinear set $u^{i} \equiv(\rho, \theta, \zeta)$ is

$$
\sqrt{g} \equiv \frac{1}{\nabla \rho \cdot \nabla \theta \times \nabla \zeta}=\frac{\partial \psi / \partial \rho}{\mathbf{B} \cdot \boldsymbol{\nabla} \theta}=\frac{\partial \psi / \partial \rho}{I / q R^{2}} \simeq r a R_{0} .
$$

The volume of a magnetic-flux surface whose label is $\rho$ is 


$$
\begin{aligned}
V(\rho, t) & \equiv \int_{0}^{\rho} d^{3} x \equiv \int_{0}^{\rho} d \rho \int_{-\pi}^{\pi} d \theta \int_{-\pi}^{\pi} d \zeta \sqrt{g} \\
& =2 \pi \int_{0}^{\rho} d \rho \int_{-\pi}^{\pi} \sqrt{g} d \theta \simeq \pi r^{2}\left(2 \pi R_{0}\right),
\end{aligned}
$$

and its radial derivative is

$$
V^{\prime} \equiv \frac{\partial V(\rho, t)}{\partial \rho}=2 \pi \int_{-\pi}^{\pi} \sqrt{g} d \theta \simeq a(2 \pi r)\left(2 \pi R_{0}\right) .
$$

The average of an axisymmetric $(\partial f / \partial \zeta=0)$ scalar function $f(\mathbf{x}, t)$ over a flux surface can be defined using a limiting process on the differential volume between a toroidal magnetic-flux surface at $\rho+\delta \rho$ and one at $\rho$ :

$$
\begin{aligned}
\langle f(\mathbf{x}, t)\rangle & \equiv \lim _{\delta \rho \rightarrow 0} \frac{\int_{\rho}^{\rho+\delta \rho} d \rho \int_{-\pi}^{\pi} d \theta \int_{-\pi}^{\pi} d \zeta \sqrt{g} f(\mathbf{x}, t)}{\int_{\rho}^{\rho+\delta \rho} d \rho \int_{-\pi}^{\pi} d \theta \int_{-\pi}^{\pi} d \zeta \sqrt{g}} \\
& =\frac{2 \pi}{V^{\prime}} \int_{-\pi}^{\pi} \sqrt{g} d \theta f(\mathbf{x}, t)=\frac{\oint d \theta \sqrt{g} f(\mathbf{x}, t)}{\oint d \theta \sqrt{g}} \\
& \simeq \int_{-\pi}^{\pi} \frac{d \theta}{2 \pi}\left[1+2 \frac{\rho a}{R_{0}} \cos \theta+\mathcal{O}\left(\epsilon^{2}\right)\right] f(\mathbf{x}, t) .
\end{aligned}
$$

This flux-surface-averaging operator is an annihilator for the parallel gradient operator $\mathbf{B} \cdot \boldsymbol{\nabla}$ :

$$
\langle\mathbf{B} \cdot \nabla f\rangle=0,
$$

for any function $f(\mathbf{x}, t)$ that is a periodic function of both $\theta$ and $\zeta$. For a similarly periodic vector field $\mathbf{A}(\mathbf{x}, t)$, the fluxsurface average of its divergence, which is defined by $\boldsymbol{\nabla} \cdot \mathbf{A}$ $\equiv \Sigma_{i}(1 / \sqrt{g})\left(\partial / \partial u^{i}\right)\left(\sqrt{g} \mathbf{A} \cdot \nabla u^{i}\right)$, becomes

$$
\langle\boldsymbol{\nabla} \cdot \mathbf{A}\rangle=\frac{1}{V^{\prime}} \frac{\partial}{\partial \rho}\left[V^{\prime}\langle\mathbf{A} \cdot \boldsymbol{\nabla} \rho\rangle\right] \equiv \frac{\partial}{\partial V}\langle\mathbf{A} \cdot \boldsymbol{\nabla} V\rangle .
$$

For the toroidal magnetic flux the relevant differential surface area is $d \mathbf{S}(\zeta) \equiv \sqrt{g} d \rho d \theta \boldsymbol{\nabla} \zeta$; hence using (9) and the fact that $I=I(\rho, t)$, the toroidal magnetic-flux density (flux per unit volume) becomes

$$
\begin{aligned}
\frac{\partial \psi_{t}}{\partial V} & \equiv \frac{1}{V^{\prime}} \frac{\partial \psi_{t}}{\partial \rho}=\frac{1}{V^{\prime}} \int_{-\pi}^{\pi} \sqrt{g} d \theta \mathbf{B} \cdot \nabla \zeta=\frac{\langle\mathbf{B} \cdot \nabla \zeta\rangle}{2 \pi} \\
& =I(\rho, t)\left\langle R^{-2}\right\rangle / 2 \pi \simeq B_{0} /\left(2 \pi R_{0}\right) .
\end{aligned}
$$

Magnetic-flux surfaces are rational or irrational depending on whether or not $q$ is the ratio of integers $(m, n)$ :

$$
q(\rho, t)\left\{\begin{array}{l}
=m / n, \quad \text { rational surface, } \\
\neq m / n, \quad \text { irrational surface. }
\end{array}\right.
$$

For finite $m, n\left(\leqslant n_{\max }\right)$ rational surfaces are a set of measure zero and radially isolated from each other while irrational surfaces are a dense set. Rational surfaces are of interest because their (helical) magnetic-field lines close on themselves after $m$ toroidal (or $n$ poloidal) transits.

The differential length $d \ell$ along magnetic-field lines can be obtained from the poloidal $(\boldsymbol{\nabla} \theta)$ projection of the equation $d \mathbf{x} / d \ell=\mathbf{B} / B$ that defines magnetic-field lines:

$$
d \ell=\frac{B}{\mathbf{B} \cdot \boldsymbol{\nabla} \theta} d \theta \simeq R_{0} q d \theta .
$$

The half-length $\ell_{*}$ of a closed magnetic-field line on a $q_{*}$ $\equiv q\left(\rho_{*}\right) \equiv m / n$ rational surface can be obtained by integrating (15) over $\theta$ from $-n \pi$ to $+n \pi$ :

$$
\ell_{*} \equiv \frac{1}{2} \int_{-n \pi}^{n \pi} \frac{B d \theta}{\mathbf{B} \cdot \boldsymbol{\nabla} \theta}=\pi \bar{R} q_{* n} \simeq \pi R_{0} q_{* n},
$$

in which

$$
\bar{R} \equiv \frac{1}{2 \pi q * \partial \psi / \partial \rho} \int_{-\pi}^{\pi} \sqrt{g} d \theta B=\frac{\langle B\rangle V^{\prime}}{4 \pi^{2} q_{*} \partial \psi / \partial \rho} \simeq R_{0} .
$$

Note that while helical field lines on rational surfaces with $n \sim n_{\max } \sim 10 \gg 1$ are quite long $\left(\gg \pi \bar{R} q_{*}\right)$, those with low $n$ $\left(\equiv n^{\circ}=1,2\right)$ are short $\left(\sim \pi \bar{R} q_{*}\right)$.

The radial distances between medium-order (e.g., $n$ $\sim 10 \gg 1$ ) rational surfaces are also of interest. To determine these, one first expands $q(\rho, t)$ in a Taylor series expansion about its value on a rational surface at $\rho=\rho_{*}$ :

$$
q(\rho, t) \simeq q_{*}+x q^{\prime}+\mathcal{O}\left(x^{2}\right), \quad \text { local } q(\rho) \text { expansion, }
$$

in which the following key quantities have been defined:

$$
\begin{aligned}
& q_{*} \equiv q\left(\rho_{*}\right)=m / n, \quad q \text { value on rational surface, } \\
& x \equiv \rho-\rho_{*}, \quad \text { distance from rational surface, } \\
& q^{\prime} \equiv \partial q /\left.\partial\right|_{\rho_{*}}, \quad \text { magnetic shear variable. }
\end{aligned}
$$

Note that all these quantities are dimensionless-because $\rho$ is a dimensionless radial coordinate. The distance to adjacent rational surfaces with $m \pm 1$ but the same $n$ is obtained from $1 / n=q-q_{*} \simeq x q^{\prime}:$

$$
\Delta \simeq 1 / n q^{\prime}, \quad \text { spacing of same } n \text { rational surfaces. }
$$

Here and henceforth, if $q^{\prime}$ is negative, it is replaced by $\left|q^{\prime}\right|$. Next, consider the distance between a $q_{*} \equiv m / n$ rational surface and the nearest rational surface with $n \leqslant n_{\max }$. Defining $q\left(\rho_{\max }\right)=m_{\max } / n_{\max }$ and expanding $q(\rho)=\left(m_{\max } n+1\right) / n_{\max } n$ in a Taylor series about $\rho=\rho_{\max }$, the separation is found to be

$$
\delta x \equiv \rho_{*}-\rho_{\max } \simeq \frac{1}{n_{\max } n q^{\prime}}, \quad \text { minimum spacing. }
$$

At a minimum in $q$ where $q^{\prime}$ vanishes, one obtains

$$
\delta x_{\min } \equiv \rho_{*}-\rho_{\max } \simeq\left(\frac{2}{n_{\max } n q^{\prime \prime}}\right)^{1 / 2}, \quad q^{\prime \prime} \equiv\left|\frac{\partial^{2} q}{\partial \rho^{2}}\right|_{\rho_{*}} .
$$

For $n_{\max } \gtrsim 10, q^{\prime} \sim 1$, and $q^{\prime \prime} \sim 1$, all these are small fractions of the minor radius: $\Delta \sim 1 / n_{\max } \ll 1$ for $n \sim n_{\max }$, and $\delta x$ $\lesssim 1 / n_{\max } \ll 1, \delta x_{\min } \lesssim 1 / \sqrt{n_{\max }}<1$.

\section{MAGNETIC-FLUX EVOLUTION EQUATIONS}

Equations for the evolution of the toroidal and poloidal magnetic fluxes are obtained from Faraday's law: 


$$
\partial \mathbf{B} / \partial t=-\boldsymbol{\nabla} \times \mathbf{E} .
$$

Taking the $d \mathbf{S}(\zeta) / d V \equiv \sqrt{g} d \theta \boldsymbol{\nabla} \zeta / V^{\prime}$ projection of this equation divided by $2 \pi$, using the definition of $\partial \psi_{t} / \partial V$ from (13), integrating over $\theta$ from $-\pi$ to $\pi$, and using common vector identities and (12), one obtains ${ }^{9}$

$$
\begin{aligned}
\left.\frac{\partial}{\partial V} \frac{\partial \psi_{t}}{\partial t}\right|_{\mathbf{x}} & =-\frac{1}{V^{\prime}} \int_{-\pi}^{\pi} \sqrt{g} d \theta \boldsymbol{\nabla} \zeta \cdot \boldsymbol{\nabla} \times \mathbf{E}=\frac{\langle\boldsymbol{\nabla} \cdot \boldsymbol{\nabla} \zeta \times \mathbf{E}\rangle}{2 \pi} \\
& =\frac{\partial}{\partial V}\left(\frac{\langle\boldsymbol{\nabla} \zeta \times \mathbf{E} \cdot \boldsymbol{\nabla} V\rangle}{2 \pi}\right) .
\end{aligned}
$$

Integrating this last form over volume from the magnetic axis where $V=\psi_{t}=0$, which remains fixed in space since $\boldsymbol{\nabla} V=\mathbf{0}$ and hence $\partial \psi_{t} / \partial t=0$ there, to $V$ yields

$$
\left.\frac{\partial \psi_{t}}{\partial t}\right|_{\mathbf{x}}=-\frac{\langle\mathbf{E} \cdot \boldsymbol{\nabla} \zeta \times \boldsymbol{\nabla} V\rangle}{2 \pi}=-\frac{\left\langle\mathbf{E} \cdot \mathbf{B}_{p}\right\rangle q}{\langle\mathbf{B} \cdot \boldsymbol{\nabla} \zeta\rangle} .
$$

Here, (13) and the fact from (5) that $\partial \psi_{t} / \partial V=q \partial \psi / \partial V$ have been used. Thus, since $\mathbf{B}_{p} \equiv \boldsymbol{\nabla} \zeta \times \nabla \psi$ is proportional to the covariant base vector in the poloidal direction, changes in the toroidal magnetic flux are induced by the (inductive) poloidal electric field in the plasma.

In a tokamak $\left\langle\mathbf{E} \cdot \mathbf{B}_{p}\right\rangle$ is mostly caused by transient changes in the radial position (Shafranov shift) and shape (ellipticity, triangularity, etc.) of magnetic-flux surfaces induced by changing poloidal currents in the external poloidal field shaping coils that produce changes in the toroidal magnetic flux. [These externally induced effects can be introduced explicitly by adding a spatially constant $\partial \Psi_{t} / \partial t$ $=V_{\text {loop }}^{\theta}(t) / 2 \pi$ term to the right of (27).] The induced motion of the toroidal flux surfaces is usually called the "grid velocity." In RFPs and spheromaks inductive poloidal electric fields are often induced transiently during "helicity injection." [There is also a small poloidal electric field induced by the parallel (to B) plasma resistivity which causes diffusion of the toroidal magnetic flux that is of order $\epsilon^{2}, B_{p}^{2} / B_{t}^{2} \ll 1$ smaller than diffusion of the poloidal magnetic flux $\psi$ and hence will be neglected-see discussion after (36) below.]

The radial grid velocity $\mathbf{u}_{g}$ of toroidal flux surfaces (relative to laboratory coordinates) can be determined from the equation obtained by setting the total differential of $\psi_{t}(\mathbf{x}, t)$ to zero $\left[d \psi_{t}=0=d \mathbf{x} \cdot \boldsymbol{\nabla} \psi_{t}+\left(\partial \psi_{t} / \partial t\right) d t\right]$ :

$$
\mathbf{u}_{g} \cdot \boldsymbol{\nabla} \psi_{t} \equiv \frac{d \mathbf{x}}{d t} \cdot \nabla \psi_{t}=-\left.\frac{\partial \psi_{t}}{\partial t}\right|_{\mathbf{x}}
$$

Flux-surface averaging and combining with (27) yields

$$
\begin{aligned}
& \left.\frac{d \psi_{t}}{d t} \equiv \frac{\partial \psi_{t}}{\partial t}\right|_{\mathbf{x}}+\left\langle\mathbf{u}_{g} \cdot \nabla \psi_{t}\right\rangle=0, \\
& \left\langle\mathbf{u}_{g} \cdot \nabla \psi_{t}\right\rangle \equiv q\left\langle\mathbf{E} \cdot \mathbf{B}_{p}\right\rangle\langle\langle\mathbf{B} \cdot \boldsymbol{\nabla} \zeta\rangle, \quad \text { grid velocity. }
\end{aligned}
$$

Hence, the toroidal flux $\psi_{t}$ is advected radially by the grid velocity $\mathbf{u}_{g}$, but conserved in a Lagrangian frame moving with $\mathbf{u}_{g}$. Using the fact from (5) that $\nabla \psi_{t}=q \nabla \psi$ and the magnetic-field vector identity $\mathbf{B}_{p} \equiv \boldsymbol{\nabla} \zeta \times \nabla \psi=\mathbf{B}-I \nabla \zeta$, (30) can be rearranged to yield a relation that will be useful for the poloidal flux evolution equation:

$$
\langle\mathbf{E} \cdot \boldsymbol{\nabla} \zeta\rangle /\left\langle R^{-2}\right\rangle=\langle\mathbf{E} \cdot \mathbf{B}\rangle /\langle\mathbf{B} \cdot \boldsymbol{\nabla} \zeta\rangle-\left\langle\mathbf{u}_{g} \cdot \nabla \psi\right\rangle .
$$

To develop an evolution equation for the poloidal flux function $\psi$, the electric field is first split into its components in and perpendicular (or cross) to the $\boldsymbol{\nabla} \zeta$ direction:

$$
\mathbf{E}=\left(\frac{\mathbf{E} \cdot \boldsymbol{\nabla} \zeta}{|\nabla \zeta|^{2}}\right) \nabla \zeta+\nabla \zeta \times \mathbf{E}_{\wedge}, \quad \mathbf{E}_{\curlywedge} \equiv \frac{\mathbf{E} \times \boldsymbol{\nabla} \zeta}{|\boldsymbol{\nabla} \zeta|^{2}}
$$

The $\mathbf{E}_{\wedge}$ component contributes to $\partial \psi_{t} / \partial t$ [see (26)], which has already been determined. The components of Faraday's law (25) in the $\zeta=$ constant plane yield

$$
\boldsymbol{\nabla} \zeta \times \boldsymbol{\nabla}\left(\frac{\partial \psi}{\partial t}-\frac{\mathbf{E} \cdot \boldsymbol{\nabla} \zeta}{|\boldsymbol{\nabla} \zeta|^{2}}\right)=\mathbf{0} .
$$

Since $|\nabla \zeta|^{2}=R^{-2}$, the solution of this equation is

$$
\frac{\partial \psi}{\partial t}=R^{2}(\mathbf{E} \cdot \nabla \zeta)-\frac{\partial \Psi}{\partial t},
$$

in which $\partial \Psi / \partial t \equiv V_{\text {loop }}^{\zeta}(t) / 2 \pi$ is a (positive) constant of the spatial integration. It represents the toroidal loop voltage induced by the rate of change of the magnetic flux in the central solenoid of a tokamak-the ohmic heating transformer. (The sign is negative because the Poynting flux usually points into the plasma-to drive the toroidal current.) Multiplying by $1 / R^{2}$, taking the flux-surface average, and then dividing by $\left\langle R^{-2}\right\rangle$ yields

$$
\frac{\partial \psi}{\partial t}=\frac{\langle\mathbf{E} \cdot \boldsymbol{\nabla} \zeta\rangle}{\left\langle R^{-2}\right\rangle}-\frac{\partial \Psi}{\partial t} .
$$

Substituting in $\langle\mathbf{E} \cdot \boldsymbol{\nabla} \zeta\rangle$ from (31) finally yields the desired evolution equation for the poloidal magnetic flux:

$$
\left.\frac{d \psi}{d t} \equiv \frac{\partial \psi}{\partial t}\right|_{\mathbf{x}}+\left\langle\mathbf{u}_{g} \cdot \boldsymbol{\nabla} \psi\right\rangle=\frac{\langle\mathbf{E} \cdot \mathbf{B}\rangle}{\langle\mathbf{B} \cdot \boldsymbol{\nabla} \zeta\rangle}-\frac{\partial \Psi}{\partial t} .
$$

The poloidal magnetic flux $\psi$ and hence field lines move relative to the toroidal flux $\psi_{t}$ [compare (29) and (36)] because of departures from ideal MHD (i.e., a nonzero parallel electric field $\langle\mathbf{E} \cdot \mathbf{B}\rangle$ ) or a temporally changing magnetic flux in the central solenoid (i.e., $\partial \Psi / \partial t \neq 0$ ). [The parallel electric field $\mathbf{E}_{\|} \equiv\langle\mathbf{E} \cdot \mathbf{B}\rangle \mathbf{B} /\left\langle B^{2}\right\rangle$ also induces [see (9.24) in Ref. 8] a small contribution to the grid velocity: $\left\langle\mathbf{u}_{g} \cdot \boldsymbol{\nabla} \psi\right\rangle\langle\mathbf{B} \cdot \boldsymbol{\nabla} \zeta\rangle$ $=q\left\langle\mathbf{E}_{\|} \cdot \mathbf{B}_{p}\right\rangle=q\langle\mathbf{E} \cdot \mathbf{B}\rangle\left(1-I^{2}\left\langle R^{-2}\right\rangle /\left\langle B^{2}\right\rangle\right) \sim \mathcal{O}\left(\epsilon^{2}, B_{p}^{2} / B_{t}^{2}\right)\langle\mathbf{E} \cdot \mathbf{B}\rangle$, which will be neglected relative to the dominant $\langle\mathbf{E} \cdot \mathbf{B}\rangle$ term on the right of (36).] Note that only the inductive electric field $\mathbf{E}^{A} \equiv-\partial \mathbf{A} / \partial t$ contributes to $d \psi / d t$-because from (11) the flux-surface average of a potential contribution vanishes: $-\langle\mathbf{B} \cdot \boldsymbol{\nabla} \phi\rangle=0$. To proceed further, plasma physics effects need to be introduced through a parallel Ohm's law that relates the parallel inductive electric field $\mathbf{E}_{\|}$to plasma resistivity times the parallel current flowing in the plasma. The resultant introduction of plasma resistivity causes the poloidal magnetic field $\mathbf{B}_{p}$ and flux $\psi$ to be a driven-dissipative magnetic-field system.

\section{PARALLEL OHM'S LAW}

A parallel Ohm's law can be obtained from the total electron momentum equation: 


$$
m_{e} n_{e} \frac{d_{e} \mathbf{V}_{e}}{d t}=-n_{e} e\left(\mathbf{E}+\mathbf{V}_{e} \times \mathbf{B}\right)-\nabla p_{e}-\boldsymbol{\nabla} \cdot \boldsymbol{\pi}_{e}+\mathbf{R}_{e} .
$$

Here, $d_{e} / d t \equiv \partial / \partial t+\mathbf{V}_{e} \cdot \boldsymbol{\nabla}, \mathbf{V}_{e}$ is the flow velocity of the electron fluid, $p_{e} \equiv n_{e} T_{e}$ is the electron pressure, $\boldsymbol{\nabla} \cdot \boldsymbol{\pi}_{e} \simeq \boldsymbol{\nabla} \cdot \boldsymbol{\pi}_{\| e}$ is the electron viscous force density due primarily to neoclassical parallel collisional stresses in the electron fluid, $\mathbf{R}_{e}$ $\simeq n_{e} e\left(\eta_{\|} \mathbf{J}_{\|}+\eta_{0} \mathbf{J}_{\perp}\right)$ is the Coulomb collision frictional force density on the electron fluid, and the other notation is standard. Taking the parallel (B.) projection of this equation, flux-surface averaging assuming $n_{e}$ is constant along $\mathbf{B}$, and using (10) and (11), the resultant equation can be rearranged to yield

$$
\langle\mathbf{E} \cdot \mathbf{B}\rangle=\eta_{\|}\langle\mathbf{J} \cdot \mathbf{B}\rangle-\frac{1}{n_{e} e}\left\langle\mathbf{B} \cdot \boldsymbol{\nabla} \cdot \boldsymbol{\pi}_{\| e}\right\rangle-\frac{m_{e}}{e}\left\langle\mathbf{B} \cdot \frac{d_{e} \mathbf{V}_{e}}{d t}\right\rangle .
$$

The first term on the right is the inductive electric field due to the parallel resistivity acting on the parallel current while the other terms represent effects due to parallel electron viscosity $^{8}$ (trapped particle effects on resistivity and bootstrap current) and electron inertia.

The inertia term can be simplified assuming that (1) the calculation is in the rest frame of the ion fluid so that $\mathbf{V}_{e}=-\mathbf{J} / n_{e} e, \quad$ (2) $\left\langle\mathbf{B} \cdot d_{e} \mathbf{V}_{e} / d t\right\rangle \simeq-\left(d_{e} / d t\right)\langle\mathbf{B} \cdot \mathbf{J}\rangle / n_{e} e \quad[$ i.e., $\left\langle\mathbf{J} \cdot d_{e} \mathbf{B} / d t\right\rangle$ is higher order and hence negligible], and (3) the electron flow velocity $\mathbf{V}_{e} \simeq \mathbf{u}_{g}$ (i.e., the classical and neoclassical electron transport flows are neglected, which is justified because the classical and neoclassical electron diffusivities ${ }^{7}$ are, respectively, factors of $\beta_{e} \equiv n_{e} T_{e} /\left(B^{2} / 2 \mu_{0}\right)$ and $\beta_{e} q^{2} / \epsilon^{3 / 2}$ smaller than the magnetic-field diffusivity $\left.D_{\eta}\right)$. Then, the inertia term becomes $\left(m_{e} / n_{e} e^{2}\right)(d / d t)\langle\mathbf{J} \cdot \mathbf{B}\rangle$ and using the definitions

$$
\begin{aligned}
& \omega_{p} \equiv \sqrt{n_{e} e^{2} / m_{e} \epsilon_{0}}, \quad \text { electron plasma frequency, } \\
& \delta_{e} \equiv c / \omega_{p}, \quad \text { electromagnetic (em) skin depth, }
\end{aligned}
$$

the parallel electric-field equation (38) can be written as

$$
\langle\mathbf{E} \cdot \mathbf{B}\rangle=\left(\frac{\eta_{\|}}{\mu_{0}}+\delta_{e}^{2} \frac{d}{d t}\right)\left\langle\mu_{0} \mathbf{J} \cdot \mathbf{B}\right\rangle-\frac{1}{n_{e} e}\left\langle\mathbf{B} \cdot \boldsymbol{\nabla} \cdot \boldsymbol{\pi}_{\| e}\right\rangle .
$$

A closure relation for the flux-surface-averaged parallel viscous force density $\left\langle\mathbf{B} \cdot \boldsymbol{\nabla} \cdot \boldsymbol{\pi}_{\| e}\right\rangle$ is calculated in neoclassical theory. ${ }^{8,13}$ It is valid on time scales after poloidal electron flows have come into equilibrium (i.e., $t>1 / \nu_{e}$-see Ref. 14) and length scales longer than the em skin depth $\left(\delta_{e}^{2} \nabla^{2}\right.$ $<1)$-so short-time-scale electron kinetics and inertial effects can be neglected. Neglecting poloidal electron heat flow and higher-order poloidal flow moment effects, the parallel electron viscous force is ${ }^{8,13}$

$$
\left\langle\mathbf{B} \cdot \boldsymbol{\nabla} \cdot \boldsymbol{\pi}_{\| e}\right\rangle \simeq m_{e} n_{e} \mu_{e} U_{\theta e}\left\langle B^{2}\right\rangle .
$$

Here, $\mu_{e}$ is the (collisional) electron viscous drag frequency [see (47) below] and

$$
U_{\theta e}(\psi) \equiv \frac{\mathbf{V}_{e} \cdot \boldsymbol{\nabla} \theta}{\mathbf{B} \cdot \boldsymbol{\nabla} \theta}=\frac{\mathbf{V}_{e} \cdot \mathbf{B}}{B^{2}}+\frac{I}{B^{2}}\left(\frac{d \Phi}{d \psi}-\frac{1}{n_{e} e} \frac{d p_{e}}{d \psi}\right)
$$

indicates the poloidal electron flow speed ${ }^{8}$ in the presence of the lowest-order parallel, $\mathbf{E} \times \mathbf{B}$ and diamagnetic electron flows. In the ion rest frame $V_{\| i}=0$ and $U_{\theta i}=0$; then, the radial ion force balance equation yields $d \Phi / d \psi=-\left(1 / n_{i} q_{i}\right)$ $\times\left(d p_{i} / d \psi\right)$. Thus, in the ion rest frame the poloidal electron current becomes

$$
-n_{e} e U_{\theta e}=(\langle\mathbf{J} \cdot \mathbf{B}\rangle+I d P / d \psi) /\left\langle B^{2}\right\rangle,
$$

in which $P \equiv p_{e}+p_{i}$ is the total plasma pressure. Substituting this last form into (42) yields

$$
-\frac{1}{n_{e} e}\left\langle\mathbf{B} \cdot \boldsymbol{\nabla} \cdot \boldsymbol{\pi}_{\| e}\right\rangle=\frac{\eta_{0}}{\mu_{0}} \frac{\mu_{e}}{\nu_{e}}\left(\left\langle\mu_{0} \mathbf{J} \cdot \mathbf{B}\right\rangle+\mu_{0} I \frac{d P}{d \psi}\right),
$$

in which the reference (perpendicular) resistivity $\eta_{0}$ is written in the form of a magnetic-field diffusivity,

$$
\frac{\eta_{0}}{\mu_{0}} \equiv \frac{m_{e} \nu_{e}}{n_{e} e^{2} \mu_{0}}=\nu_{e} \delta_{e}^{2} \simeq \frac{1.4 \times 10^{3} Z}{\left[T_{e}(\mathrm{eV})\right]^{3 / 2}}\left(\frac{\ln \Lambda}{17}\right) \frac{\mathrm{m}^{2}}{\mathrm{~s}},
$$

and the electron viscous drag frequency adapted from Refs. 7,8 , and 13 is approximately

$$
\frac{\mu_{e}}{\nu_{e}} \simeq \frac{Z+\sqrt{2}-\ln (1+\sqrt{2})}{Z\left(1+\nu_{*_{e}}^{1 / 2}+\nu_{*_{e}}\right)} \frac{f_{t}}{f_{c}} \stackrel{\substack{\nu_{*}=0 \\ Z=1}}{\Rightarrow} 1.5 \frac{f_{t}}{f_{c}} .
$$

In these formulas, $Z\left(\rightarrow Z_{\mathrm{eff}} \equiv \sum_{i} n_{i} Z_{i}^{2} / n_{e}\right.$ for multiple ion species) is the (effective) ion charge, $f_{c}$ is the flow-weighted fraction of circulating particles ${ }^{8}$ for which a Padé approximation is ${ }^{15}$

$$
f_{c} \simeq \frac{\left(1-\epsilon^{2}\right)^{-1 / 2}(1-\epsilon)^{2}}{1+1.46 \epsilon^{1 / 2}+0.2 \epsilon} \simeq 1-1.46 \epsilon^{1 / 2}+\mathcal{O}(\epsilon) .
$$

Also, the fraction of trapped particles is defined by $f_{t} \equiv 1$ $-f_{c}$ and the electron collisionality parameter is

$$
\nu_{*_{e}} \equiv \frac{\nu_{e}}{\epsilon^{3 / 2}\left(v_{T_{e}} / \bar{R} q\right)}=\frac{\bar{R} q}{\epsilon^{3 / 2} \lambda_{e}}, \quad v_{T_{e}} \equiv \sqrt{\frac{2 T_{e}}{m_{e}}},
$$

in which the electron collision length $\lambda_{e}$ is given by

$$
\lambda_{e} \equiv \frac{v_{T_{e}}}{\nu_{e}} \simeq 1.2 \times 10^{16} \frac{\left[T_{e}(e V)\right]^{2}}{n_{e} Z}\left(\frac{17}{\ln \Lambda}\right) \mathrm{m} .
$$

Since the parallel viscous force $\left\langle\mathbf{B} \cdot \boldsymbol{\nabla} \cdot \boldsymbol{\pi}_{\| e}\right\rangle$ has a term proportional to $\left\langle\mu_{0} \mathbf{J} \cdot \mathbf{B}\right\rangle$, when it is introduced in (41) this term just adds to the parallel resistivity term. Thus, upon substituting the closure (45) into (41) one obtains

$$
\langle\mathbf{E} \cdot \mathbf{B}\rangle=\left(\frac{\eta_{\|}^{\mathrm{nc}}}{\mu_{0}}+\delta_{e}^{2} \frac{d}{d t}\right)\left\langle\mu_{0} \mathbf{J} \cdot \mathbf{B}\right\rangle+\frac{\mu_{e}}{\nu_{e}} \eta_{0} I \frac{d P}{d \psi},
$$

in which

$$
\eta_{\|}^{\mathrm{nc}} \equiv \eta_{0}\left(\frac{\eta_{\|}^{\mathrm{Sp}}}{\eta_{0}}+\frac{\mu_{e}}{\nu_{e}}\right), \quad \text { neoclassical resistivity }
$$

and the parallel $\left(\right.$ Spitzer $^{8,16}$ ) electrical resistivity is approximately (including electron flow, heat flow effects) 


$$
\frac{\eta_{\|}^{\mathrm{Sp}}}{\eta_{0}} \simeq \frac{\sqrt{2}+Z}{\sqrt{2}+13 Z / 4}, \quad \text { Spitzer resistivity }
$$

This last formula is accurate (for all $Z$ ) to about $5 \%$, which is within the intrinsic accuracy $(\sim 1 / \ln \Lambda \sim 1 / 17 \sim 6 \%)$ of the Fokker-Planck collision operator used to obtain it. The $\eta_{\|}^{\text {nc }}$ in (52) ranges from being equal (for $\mu_{e} / \nu_{e} \ll 1$ ) to twice as large as (for $\mu_{e} / \nu_{e} \gg 1$ ), the most precise neoclassical resistivity results. ${ }^{7,17}$

Next, $\left\langle\mu_{0} \mathbf{J} \cdot \mathbf{B}\right\rangle$ is obtained. Using Ampere's law, the current density $\mathbf{J}$ embodied in the $\mathbf{B}$ defined in (3) is ${ }^{11}$

$$
\mu_{0} \mathbf{J} \equiv \boldsymbol{\nabla} \times \mathbf{B}=(\partial I / \partial \psi) \nabla \psi \times \nabla \zeta+\nabla \zeta \Delta^{*} \psi,
$$

in which $\Delta^{*} \psi$ is the usual magnetic differential operator:

$$
\Delta^{*} \psi \equiv\left(1 /|\boldsymbol{\nabla} \zeta|^{2}\right) \boldsymbol{\nabla} \cdot|\boldsymbol{\nabla} \zeta|^{2} \boldsymbol{\nabla} \psi=R^{2} \boldsymbol{\nabla} \cdot R^{-2} \boldsymbol{\nabla} \psi .
$$

Dotting this $\mu_{0} \mathbf{J}$ with $\mathbf{B}$ from (3), flux-surface averaging, and using $|\boldsymbol{\nabla} \zeta|^{2}=R^{-2}$ and (11), yields [see Eq. (7.20) and its antecedents in Ref. 7],

$$
\begin{aligned}
\left\langle\mu_{0} \mathbf{J} \cdot \mathbf{B}\right\rangle & =I\left\langle|\nabla \zeta|^{2} \Delta^{*} \psi\right\rangle-(\partial I / \partial \psi)\left\langle|\nabla \zeta \times \nabla \psi|^{2}\right\rangle \\
& =I \frac{\partial}{\partial V}\left\langle\frac{\boldsymbol{\nabla} V \cdot \boldsymbol{\nabla} \psi}{R^{2}}\right\rangle-\frac{\partial I}{\partial \psi}\left\langle\frac{|\boldsymbol{\nabla} \psi|^{2}}{R^{2}}\right\rangle \\
& \equiv\langle\mathbf{B} \cdot \boldsymbol{\nabla} \zeta\rangle \Delta^{+} \psi=I\left\langle R^{-2}\right\rangle \Delta^{+} \psi,
\end{aligned}
$$

in which for notational simplicity

$$
\Delta^{+} \psi \equiv \frac{I}{\left\langle R^{-2}\right\rangle V^{\prime}} \frac{\partial}{\partial \rho}\left[\left\langle\frac{|\nabla \rho|^{2}}{R^{2}}\right\rangle \frac{V^{\prime}}{I} \frac{\partial \psi}{\partial \rho}\right] \simeq \frac{1}{r} \frac{\partial}{\partial r} r \frac{\partial \psi}{\partial r} .
$$

Note that $\Delta^{+}$is a second-order Laplacian-type differential operator on the poloidal magnetic flux $\psi$, which in the large aspect ratio tokamak limit becomes the radial part of a cylindrical $\nabla^{2}$ operator.

Substituting the expression for $\left\langle\mu_{0} \mathbf{J} \cdot \mathbf{B}\right\rangle$ from (56) in (51) and dividing by $\langle\mathbf{B} \cdot \nabla \zeta\rangle=I\left\langle R^{-2}\right\rangle$, one finally obtains the desired parallel Ohm's law in the form needed for the poloidal flux evolution equation (36):

$$
\frac{\langle\mathbf{E} \cdot \mathbf{B}\rangle}{\langle\mathbf{B} \cdot \nabla \zeta\rangle}=\left(\frac{\eta_{\|}^{\mathrm{nc}}}{\mu_{0}}+\delta_{e}^{2} \frac{d}{d t}\right) \Delta^{+} \psi+\frac{\mu_{e}}{\nu_{e}} \eta_{0} \frac{1}{\left\langle R^{-2}\right\rangle} \frac{d P}{d \psi} .
$$

Here, the first term indicates magnetic-flux diffusion (see Sec. V) induced by the neoclassical parallel resistivity, the second is due to electron inertia, and the last is caused by the neoclassical bootstrap current which for $\nu_{*_{e}}=0$ becomes $1.5\left(f_{t} / f_{c}\right) \eta_{0}\left(R_{0} / B_{p}\right)(d P / d r) \sim 1.1 \sqrt{\epsilon}\left(d \beta_{p} / d r\right)\left(\eta_{0} / \mu_{0}\right)\left(B_{p} R_{0}\right)$ in the large aspect ratio tokamak limit. If an extra, noninductive force on electrons $\mathbf{F}_{e}$ is added to the electron momentum equation (37), it would induce an additional "source" current $\left\langle\mathbf{J}_{S} \cdot \mathbf{B}\right\rangle \equiv-\left\langle\mathbf{F}_{e} \cdot \mathbf{B}\right\rangle /\left(n_{e} e \eta_{\|}^{\mathrm{nc}}\right)$, and hence an extra contribution of $-\eta_{\|}^{\text {nc }}\left\langle\mathbf{J}_{S} \cdot \mathbf{B}\right\rangle /\langle\mathbf{B} \cdot \nabla \zeta\rangle$ on the right of (58).

\section{MAGNETIC-FLUX, FIELD-LINE DIFFUSION}

Magnetic-field lines can be defined and do not change their topology in ideal MHD. Using a Clebsch representation $\mathbf{B}=\boldsymbol{\nabla} \alpha \times \boldsymbol{\nabla} \beta$, one first notes that the local direction of $\mathbf{B}$ is given by the cross product of the gradients of $\alpha$ and $\beta$, which are perpendicular to the $\alpha$ and $\beta$ surfaces. Hence, a magnetic-field line lies at the intersection of $\alpha=$ constant $_{1}$ and $\beta=$ constant $_{2}$ surfaces. Next, using the Clebsch representation and the ideal MHD Ohm's law $\mathbf{E}+\mathbf{V} \times \mathbf{B}=\mathbf{0}$ in Faraday's law (25), one can show that

$$
d \alpha / d t=0, \quad d \beta / d t=0 \quad \text { with } d / d t \equiv \partial / \partial t+\mathbf{V} \cdot \nabla .
$$

Since $\alpha$ and $\beta$ do not change in time on a given magneticfield line, even as it moves, they provide labels to define it in the plane perpendicular to $\mathbf{B}$ in ideal MHD. A convenient third coordinate in the Clebsch representation is the length $\ell$ along a magnetic-field line which is determined from the projection of the equation for a magnetic-field line $(d \mathbf{x} / d \ell$ $=\mathbf{B}(B)$ in a desired direction.

For the axisymmetric magnetic-field model in (3), one can identify the Clebsch coordinates as $\alpha \rightarrow \psi$ and $\beta \rightarrow q \theta$ $-\zeta$; the length $\ell$ along magnetic-field lines is determined from (15). However, there is a well-known problem with Clebsch coordinates on irrational magnetic-field lines: when $q$ is not the ratio of integers, the field-line label $\beta \equiv q \theta-\zeta$ is not periodic in $\theta$.

This periodicity difficulty will be addressed by introducing a local coordinate system in the vicinity of a given rational surface at $\rho=\rho_{*}$ where $q_{*} \equiv q\left(\rho_{*}\right)=m / n$. Namely, a new angular coordinate is introduced: ${ }^{18}$

$$
\alpha \equiv \zeta-q_{*} \theta=\zeta-(m / n) \theta, \quad \text { helical angle. }
$$

Note that $\nabla \alpha$ is perpendicular (at all $\rho$ ) to the helical trajectory of a $q_{*}$ rational field line. Since $\boldsymbol{\nabla} \theta \times \boldsymbol{\nabla} q_{*} \theta=\mathbf{0}$, the Jacobian $\sqrt{g} \equiv(\boldsymbol{\nabla} \rho \cdot \boldsymbol{\nabla} \theta \times \boldsymbol{\nabla} \alpha)^{-1}$ for transforming from the fixed Eulerian grid $\mathbf{x}$ to the coordinates $\rho, \theta, \alpha$ is the same as before and thus given by (7). Writing $\mathbf{B}$ in (3) as $\mathbf{B}=\boldsymbol{\nabla} \psi$ $\times \nabla\left[\left(q_{*} \theta-\zeta\right)+\left(q-q_{*}\right) \theta\right]$, it can be rewritten in terms of these new coordinates as a helical component with the pitch of the rational field line $\left(\mathbf{B}_{h}\right)$ plus a magnetic-shear-induced component $\left(\mathbf{B}_{*}\right)$ :

$$
\begin{aligned}
& \mathbf{B}=\boldsymbol{\nabla} \alpha \times \boldsymbol{\nabla} \psi+\boldsymbol{\nabla} \psi * \nabla \theta \equiv \mathbf{B}_{h}+\mathbf{B}_{*}=\boldsymbol{\nabla} \times \mathbf{A} \\
& \text { for } \mathbf{A} \equiv-\psi \nabla \alpha+\psi * \boldsymbol{\nabla} \theta .
\end{aligned}
$$

Here, the helical magnetic flux $\psi *$ is defined by

$$
\frac{\partial \psi_{*}}{\partial \rho}=\left(q-q_{*}\right) \frac{\partial \psi}{\partial \rho}, \quad \text { helical flux definition } .
$$

It can be integrated across a rational surface to yield

$$
\psi_{*}(\rho, t)=\int_{\rho_{*}}^{\rho} d \rho \frac{\partial \psi}{\partial \rho}\left(q-q_{*}\right)=\int_{\psi_{t}\left(\rho_{*}\right)}^{\psi_{t}(\rho, t)} d \psi_{t}-q_{*} \int_{\psi\left(\rho_{*}\right)}^{\psi(\rho, t)} d \psi .
$$

Using the Taylor series expansion of $q(\rho)$ about the rational surface in (18), one obtains from (63)

$$
\psi_{*} \simeq\left(x^{2} / 2\right) q^{\prime} \psi^{\prime}+\mathcal{O}\left(x^{3}\right), \text { helical flux. }
$$

Here, as in (21) $q^{\prime} \equiv \partial q /\left.\partial \rho\right|_{\rho_{*}}$ and $\psi^{\prime} \equiv \partial \psi /\left.\partial \rho\right|_{\rho_{*}}$. The helical flux function can also be defined by the relation $\psi_{*}$ $\equiv \iint d \mathbf{S}(\alpha) \cdot \mathbf{B}=\int_{\rho_{*}}^{\rho} d \rho \int_{-\pi}^{\pi} d \theta \sqrt{g} \boldsymbol{\nabla} \alpha \cdot \mathbf{B}$.

The introduction of plasma resistivity leads to radial diffusion of magnetic-field lines. In Sec. III it was shown that 
while the toroidal magnetic flux $\psi_{t}$ does not change in time [in the Lagrangian frame moving with the grid velocity $\mathbf{u}_{g}$ defined in (30)], the poloidal magnetic flux does change if there is an electric-field component parallel to the magnetic field B. Substituting the Ohm's-law-determined parallel electric field in (58) into the poloidal flux evolution equation (36), one obtains the diffusion-type (at least for $\delta_{e}^{2} \Delta^{+} \ll 1$ ) equation

$$
\frac{d}{d t}\left(1-\delta_{e}^{2} \Delta^{+}\right) \psi=D_{\eta} \Delta^{+} \psi-S_{\psi} .
$$

Here, the diffusivity for the poloidal magnetic flux (and hence for poloidal magnetic-field lines) is

$$
D_{\eta} \equiv \eta_{\|}^{\mathrm{nc}} / \mu_{0}, \quad \text { magnetic-field diffusivity, }
$$

and the source $S_{\psi}$ of poloidal magnetic flux is

$$
S_{\psi} \equiv \frac{\partial \Psi}{\partial t}-\frac{\mu_{e}}{\nu_{e}} \eta_{0} \frac{1}{\left\langle R^{-2}\right\rangle} \frac{d P}{d \psi} .
$$

The two sources of poloidal flux are due to the "currentdrive" effects of the magnetic-flux change in the central solenoid (first term: $\partial \Psi / \partial t \equiv V_{\text {loop }}^{\zeta} / 2 \pi$ ) and the bootstrap current (second term); both contribute positively to $S_{\psi}$ for the usual confined plasma situation where $d P / d \psi<0$. An extra, noninductive current source $\mathbf{J}_{S}$ would add a source term $\eta_{\|}^{\text {nc }}\left\langle\mathbf{J}_{S} \cdot \mathbf{B}\right\rangle / I\left\langle R^{-2}\right\rangle$ to (67).

For scale lengths longer than the em skin depth $\delta_{e}$ (i.e., for $\delta_{e}^{2} \Delta^{+} \ll 1$ ), the poloidal flux evolution equation (65) becomes a diffusion (parabolic) equation for $\psi$. Comparing this diffusion equation with (29), one sees that the poloidal flux $\psi$ and hence bundles of poloidal magnetic-field lines diffuse radially (relative to the toroidal flux $\psi_{t}$, field lines). Note from $D_{\eta} \equiv \eta_{\|}^{\mathrm{nc}} / \mu_{0}$ and the definition of $\Delta^{+} \psi$ in (56) that the necessary conditions for diffusion of poloidal flux are that there must be parallel resistivity and a flux-surface-averaged parallel current $\langle\mathbf{J} \cdot \mathbf{B}\rangle$ flowing in the plasma (i.e., $\eta_{\|}\langle\mathbf{J} \cdot \mathbf{B}\rangle$ $\neq 0$ ).

Consider first the equilibrium solution of (65) for the poloidal magnetic flux $\psi$. For equilibrium in the Lagrangian frame, $d / d t \rightarrow 0$ and the equation for the stationary poloidal flux $\psi_{0}$ becomes

$$
0=D_{\eta} \Delta^{+} \psi_{0}-S_{\psi}, \quad \psi \text { equilibrium. }
$$

Thus, in equilibrium the diffusion of $\psi$ (and hence of poloidal field lines) is balanced by the source $S_{\psi}$ of the poloidal magnetic-flux, field lines; the Poynting flux represented by $\partial \Psi / \partial t$ brings poloidal flux into the plasma and the magneticfield diffusivity $D_{\eta}$ diffuses it out of the plasma-even for a stationary poloidal magnetic field. The combination of the source $S_{\psi}$ and the magnetic-field diffusion of poloidal flux (i.e., $D_{\eta} \Delta^{+} \psi_{0} \propto \eta_{\|}^{\text {nc }}\langle\mathbf{J} \cdot \mathbf{B}\rangle \neq 0$ ) causes the poloidal magnetic field to be a driven-dissipative system on the resistive or "skin" time scale $\tau_{\eta} \sim a^{2} / 6 D_{\eta}$ in current-carrying toroidal plasmas.

Diffusion of a quantity balancing a source of it is typical of any diffusive process in stationary equilibrium. For example, the density evolution equation with a density source $S_{n}$ and Fick's diffusion law $\boldsymbol{\Gamma} \equiv n \mathbf{V}=-D \boldsymbol{\nabla} n$ becomes $\partial n / \partial t$
$=D \nabla^{2} n+S_{n}$; in equilibrium this yields $0=D \nabla^{2} n+S_{n}$. (The sign of the density source $S_{n}$ is opposite to $S_{\psi}$ because while $\psi$ increases with radius, $n$ usually decreases with radius; hence, $\Delta^{+} \psi>0$ while $\nabla^{2} n<0$.) Fick's law for the radial diffusive "flux" of poloidal magnetic flux $\psi$ is $\Gamma_{\psi}$ $\simeq-D_{\eta} d \psi / d r$ in a large aspect ratio tokamak with uniform resistivity.

Physically, an initially localized poloidal flux spreads radially in time via diffusion. A mathematical description of the diffusive spreading on short time scales can be developed by writing the poloidal flux in equilibrium $\left(\psi_{0}\right)$ and localized transient $\left[\delta \psi(x, t)\right.$ for which $\left.\bar{a}^{2} \Delta^{+} \delta \psi \simeq \partial^{2} \delta \psi / \partial x^{2} \gg \delta \psi\right]$ parts: $\psi=\psi_{0}+\delta \psi$. Substituting this Ansatz into (65) and taking account of the equilibrium equation (68), one obtains (for $\left.x^{2} \ll 1\right)$

$$
\left(\frac{\partial}{\partial t}+\bar{u}_{g} \frac{\partial}{\partial x}\right)\left(1-\bar{\delta}_{e}^{2} \frac{\partial^{2}}{\partial x^{2}}\right) \delta \psi \simeq \bar{\nu}_{e} \bar{\delta}_{e}^{2} \frac{\partial^{2} \delta \psi}{\partial x^{2}},
$$

in which the following normalized variables have been introduced:

$$
\begin{aligned}
& \bar{u}_{g} \equiv\left\langle\mathbf{u}_{g} \cdot \nabla \rho\right\rangle, \quad \text { normalized grid velocity }\left(\mathrm{s}^{-1}\right), \\
& \bar{\delta}_{e} \equiv \delta_{e} / \bar{a}, \quad \text { normalized em skin depth }(\ll 1), \\
& \frac{1}{\bar{a}^{2}} \equiv \frac{1}{\left\langle R^{-2}\right\rangle}\left\langle\frac{|\nabla \rho|^{2}}{R^{2}}\right\rangle \simeq \frac{1}{a^{2}}, \quad \text { effective radius, } \\
& \bar{D}_{\eta} \equiv D_{\eta} / \bar{a}^{2}, \quad \text { normalized diffusivity }\left(\mathrm{s}^{-1}\right), \\
& \bar{\nu}_{e} \equiv \nu_{e}\left(\eta_{\|}^{\mathrm{nc}} / \eta_{0}\right), \quad \text { effective neoclassical } \nu_{e} .
\end{aligned}
$$

Properties of solutions of (69) on the $1 / \bar{\nu}_{e}$ time scale can be explored by taking a Fourier transform in the $x$ (radial) variable and neglecting (for simplicity) the grid velocity term $\bar{u}_{g} \partial / \partial x$ to yield

$$
\frac{\partial \delta \hat{\psi}}{\partial t}=-\bar{\nu}_{e} \frac{k_{x}^{2} \bar{\delta}_{e}^{2}}{1+k_{x}^{2} \bar{\delta}_{e}^{2}} \delta \hat{\psi} .
$$

Here, $\delta \hat{\psi}\left(k_{x}, t\right)$ is the Fourier transform of $\delta \psi(x, t)$. On radial scale lengths shorter than the em skin depth $\left(|x|^{2} \ll \bar{\delta}_{e}^{2}, k_{x}^{2} \bar{\delta}_{e}^{2}\right.$ $\gg 1$ ), (75) yields $\partial \delta \hat{\psi} / \partial t \simeq-\bar{\nu}_{e} \delta \hat{\psi}$, whose solution $\delta \hat{\psi}$ $=\left.\delta \hat{\psi}\right|_{t=0}\left(1-e^{-\bar{\nu}_{e} t}\right)$ approaches a constant for $t>1 / \bar{\nu}_{e}$. Physically, the poloidal flux within the em skin depth layer is spatially constant because magnetic-field lines are not diffusing there; instead, since this is a "reactive" region because the electrical resistivity is imaginary there, the poloidal magnetic field associated with $\delta \psi, \delta \mathbf{B}_{p} \equiv \boldsymbol{\nabla} \zeta \times \boldsymbol{\nabla} \delta \psi$, vanishes there $\left(|x|^{2}<\bar{\delta}_{e}^{2}\right)$.

For radial scale lengths longer than the em skin depth $\delta_{e}$ (for which $k_{x}^{2} \bar{\delta}_{e}^{2} \ll 1$ so that $\bar{\delta}_{e}^{2} \partial^{2} / \partial x^{2}$ can be neglected compared to unity), the evolution equation (69) for a localized poloidal magnetic flux $\delta \psi$ becomes 


$$
\frac{d \delta \psi}{d t} \equiv\left(\frac{\partial}{\partial t}+\bar{u}_{g} \frac{\partial}{\partial x}\right) \delta \psi=\bar{D}_{\eta} \frac{\partial^{2} \delta \psi}{\partial x^{2}} .
$$

The Green-function-type solution of this diffusion equation for a $\delta \psi$ initially radially localized at $x=x_{0}\left(>\bar{\delta}_{e}\right)$, represented by $\delta \psi(x, t=0)=\delta \psi_{0} \delta\left(x-x_{0}\right)$, is (for $\left.t \geqslant 0\right)$

$$
\delta \psi(x, t)=\delta \psi_{0} \frac{e^{-\left(x-x_{0}-\bar{u}_{t} t\right)^{2} / 4 \bar{D}_{\eta} t}}{\sqrt{4 \pi \bar{D}_{\eta} t}} .
$$

From the discussion in the preceding paragraphs, this solution is valid for $1 \gg x^{2}>\bar{\delta}_{e}^{2}$. Note that $\delta \psi$ provides a temporally evolving probability distribution for the radial location of the poloidal flux $\delta \psi_{0}$ that was initially at $x=x_{0}$ (i.e., at $\rho$ $=\rho_{*}+x_{0}$, which represents an arbitrary radial position for the present poloidal flux discussion) as it is advected (moved) radially with the grid velocity $\bar{u}_{g}$ and diffused radially due to $D_{\eta}$ in a time $t$.

The average radial displacement and spread (variance) of the initially localized flux grow linearly with time:

$$
\begin{aligned}
& \frac{\int_{-\infty}^{\infty} d x\left(x-x_{0}\right) \delta \psi}{\int_{-\infty}^{\infty} d x \delta \psi}=\bar{u}_{g} t, \\
& \frac{\int_{-\infty}^{\infty} d x\left(x-x_{0}\right)^{2} \delta \psi}{\int_{-\infty}^{\infty} d x \delta \psi}=2 \bar{D}_{\eta} t=\left(2 \bar{\nu}_{e} t\right) \bar{\delta}_{e}^{2} .
\end{aligned}
$$

Thus, poloidal flux or bundles of poloidal field lines are advected radially at speed $\bar{u}_{g}$ and diffused radially a root-meansquare-distance $x_{\mathrm{rms}}=\bar{\delta}_{e}\left(2 \bar{\nu}_{e} t\right)^{1 / 2}$ in a time $t$.

This poloidal flux (field line) diffusion process is analogous to how a group of "marked particles" would spread diffusively away from some posited initial position in the density evolution equation analogy discussed in the next full paragraph after (68). In the paleoclassical model the "marked particles" will be the small amount of poloidal magnetic flux traversed by an electron gyro-orbit and the locally Maxwellianized distributions of electrons and hence equilibrated electron temperatures on these field lines. In Sec. VI a Fokker-Planck formalism will be developed to include the effects of the radial advection and diffusion of poloidal magnetic flux on the electron guiding center positions and hence on the electron distribution function in a kinetic analysis. Note that this advection and diffusion process occurs even when the poloidal magnetic field is in stationary equilibrium (i.e., $d \psi / d t=0$ ), just as marked particles diffuse away from an initial position in the equilibrium density analogy discussed in the next full paragraph after (68).

Implicitly, the preceding analysis assumed the poloidal magnetic flux is in stationary equilibrium (at least in a Lagrangian frame moving with the grid velocity $\bar{u}_{g}$ ), i.e., $d \psi / d t=0$, as used to obtain (68). When the poloidal flux is not stationary, one can define (neglecting $\bar{u}_{g}$ )

$$
\dot{\psi} \equiv \partial \psi / \partial t=D_{\eta} \Delta^{+} \psi-S_{\psi}
$$

By analogy with identifying $\partial \Psi / \partial t$ [after (34)] as the externally applied loop voltage, $\dot{\psi}$ represents the local change in "Lenz's law" voltage on a given flux surface in the plasma: $\delta V^{\zeta}(\rho, t) \equiv \oint d \ell(\zeta) \cdot \delta \mathbf{E}=2 \pi \dot{\psi} \quad$ from $\quad \delta \mathbf{E} \equiv-\partial \delta \mathbf{A} / \partial t \quad$ and $\delta \mathbf{A}=-t \dot{\psi} \nabla \zeta$.

When $\dot{\psi} \neq 0$, the poloidal flux is changing in the plasma. This causes the $x$ position of a poloidal flux surface to move (relative to $\psi_{t}$ ). To determine this motion, the poloidal magnetic flux $\psi(\rho, t)$ can be expanded in $x$ and $t$ (assuming the flux changes slowly, i.e., $\left.\dot{\psi} \ll \bar{\nu}_{e} \psi_{0}\right)$ :

$$
\psi(x, t) \simeq \psi(0,0)+x \psi^{\prime}+t \dot{\psi}+\cdots .
$$

Setting the total derivative of this expansion to zero [to remain on the same flux surface as $\psi$ changes - see (59)], one obtains the radial speed of a field line induced by $\dot{\psi}$ :

$$
\bar{u}_{\dot{\psi}}=\frac{d x}{d t} \equiv-\frac{\dot{\psi}}{\psi^{\prime}}=-\frac{\partial \psi / \partial t}{\partial \psi / \partial \rho}, \quad u_{\dot{\psi}}=\bar{a} \bar{u}_{\dot{\psi}} .
$$

In Sec. VI the effects of the motion of poloidal magnetic flux will be characterized (in a kinetic theory context) by a Fokker-Planck ${ }^{19,20}$ description. The relevant Fokker-Planck coefficients for the radial advection and diffusion of the poloidal flux can be defined by

$$
\begin{aligned}
& \frac{\langle\Delta x\rangle}{\Delta t} \equiv \int_{-\infty}^{\infty} d x\left(x-x_{0}\right) W\left(x_{0} ; x\right), \\
& \frac{\left\langle(\Delta x)^{2}\right\rangle}{\Delta t} \equiv \int_{-\infty}^{\infty} d x\left(x-x_{0}\right)^{2} W\left(x_{0} ; x\right),
\end{aligned}
$$

in which the "transition probability" $W\left(x_{0} ; x\right)$ is the probability per unit time that a poloidal field line (infinetesimal amount of poloidal flux) will move from $x_{0}$ to $x$, for small departures of $x$ from $x_{0}:\left|x-x_{0}\right| \ll 1$. Note that the angular brackets in Fokker-Planck theory indicate ensemble average or "expectation" values-not the magnetic-flux-surface average in (10).

For the poloidal flux (field line) evolution, the appropriate transition probability is given by $W\left(x_{0} ; x\right)$ $=\partial\left[\delta \psi(x, t) / \delta \psi_{0}\right] / \partial t$. Thus, taking account of the effects in the preceding paragraphs, the motion and diffusive spread of the poloidal flux (field lines) can be written in terms of Fokker-Planck coefficients as

$$
\frac{\left\langle\Delta x_{\psi}\right\rangle}{\Delta t}=\bar{u}_{g}+\bar{u}_{\psi}, \quad \frac{\left\langle\left(\Delta x_{\psi}\right)^{2}\right\rangle}{\Delta t}=2 \bar{D}_{\eta} .
$$

These results are applicable only outside the thin em skin depth layer around the rational surface, i.e., for $|x|>\bar{\delta}_{e}$; inside (i.e., for $|x|<\bar{\delta}_{e}$ ) the coefficients vanish.

In Sec. VI the effects of magnetic-field diffusion will be considered in the vicinity of a rational surface. Thus, the motion and diffusion of the local helical flux (field lines) need to be considered. Physically, since from (63) the helical flux depends on the toroidal flux $\psi_{t}$, which does not move (in the Lagrangian frame), and the poloidal flux $\psi$, which is advected and diffused radially, one can anticipate that the localized helical flux will mostly be advected and diffused just as poloidal flux is.

To explore the behavior of the helical flux, one begins by taking the time derivative of the definition of $\psi *$ in (63) using 
$d \psi_{t} / d t=0$ from (29) and (65), to obtain [neglecting em skin depth effects for $x^{2}>\bar{\delta}_{e}^{2}$ by an analysis similar to the discussion in the paragraph following (75)]

$$
d \psi * / d t=-q_{*} d \psi / d t=-q_{*}\left(D_{\eta} \Delta^{+} \psi-S_{\psi}\right) .
$$

Next, the last definition of $\psi_{*}$ in (63) is used to write

$$
\Delta^{+} \psi=\Delta^{+}\left(-\psi_{*}+\psi_{t}\right) / q_{*} .
$$

Close to a rational surface the local radial coordinate $x$ defined in (20) is small and the $\Delta^{+}$differential operators on $\psi *$ and $\psi_{t}$ can be approximated by $\Delta^{+} \psi_{*} \simeq\left(1 / \bar{a}^{2}\right)\left(\partial^{2} \psi_{*} / \partial x^{2}\right)$ and $\Delta^{+} \psi_{t} \simeq q_{*} \Delta^{+} \psi+q^{\prime} \psi^{\prime} / \bar{a}^{2}$, which were obtained using the Taylor series expansion $d \psi_{t} / d \rho=q \partial \psi / \partial \rho \simeq\left(q_{*}+x q^{\prime}+\cdots\right) \partial \psi / \partial \rho$. Using these results and writing the helical flux in terms of equilibrium and localized transient parts $\left[\psi_{*}=\psi_{* 0}+\delta \psi_{*}(x, t)\right.$ where from (63) $\psi_{* 0} \simeq\left(x^{2} / 2\right) q^{\prime} \psi^{\prime}$ so that $\left.\Delta^{+} \psi_{* 0} \simeq q^{\prime} \psi^{\prime} / \bar{a}^{2}\right]$, one obtains from (84) and (85) (for $\bar{\delta}_{e}^{2}<x^{2} \ll 1$ )

$$
\left(\frac{\partial}{\partial t}+\bar{u}_{g} \frac{\partial}{\partial x}\right) \delta \psi_{*} \simeq \bar{D}_{\eta} \frac{\partial^{2} \delta \psi_{*}}{\partial x^{2}}-q_{*} \dot{\psi}
$$

The differential operators and Green-function-type solution for $\delta \psi_{*}$ are the same as those in (76) and (77). Thus, helical magnetic-field lines with $q_{*} \equiv m / n$ are also advected with velocity $\bar{u}_{g}$ and spread diffusively with diffusivity $\bar{D}_{\eta}$, as indicated in (78) and (79).

When the poloidal magnetic field is not in stationary equilibrium (i.e., $\dot{\psi} \neq 0$ ), the radial position of the rational surface changes. Setting the time derivative of the $q$ in (5) to zero using $0=d q_{*} / d t=\dot{\rho}_{*} q^{\prime}-\left(q_{*} / \psi^{\prime}\right)(\partial \dot{\psi} / \partial \rho)$, one finds the rational surface motion induced by $\dot{\psi}$ is $\dot{\rho}_{*}=\left(q_{*} / q^{\prime}\right)$ $\times(\partial \dot{\psi} / \partial \rho) /\left.(\partial \psi / \partial \rho)\right|_{\rho_{*}}$. Also, the helical flux changes according to $d \delta \psi_{*} / d t=-q_{*} \dot{\psi}$. Expanding such an evolving helical flux about a (moving) rational surface as in (81), one obtains $\psi_{*}(x, t) \simeq \psi_{*}(0,0)+\left(x^{2} / 2\right) q^{\prime} \psi^{\prime}-t q_{*} \dot{\psi}+\cdots$. Setting the total derivative of this helical flux function to zero (to find the motion required to stay on the same helical field line), one finds [analogously to (82)] $d\left(x^{2} / 2\right) / d t=q_{*} \dot{\psi} / q^{\prime} \psi^{\prime}$. Unlike the advective velocity $\bar{u}_{\psi}$ of poloidal field lines induced by $\dot{\psi} \neq 0$, for helical field lines $\dot{\psi} \neq 0$ induces a quadratic spreading (for $\dot{\psi}>0$ ) or contraction $(\dot{\psi}<0)$ motion-because $\psi_{*} \sim x^{2}$. However, it is not a diffusive (i.e., Markov ${ }^{19,20}$ ) process. Since these effects are rather complicated, for simplicity they will be circumvented here by assuming the induced transients are so weak that a rational surface moves less than the $\psi$ surface (i.e., $\left|\dot{\rho}_{*}\right| \ll\left|\bar{u}_{\psi}\right|$ ) and diffusion of helical field lines dominates over $\dot{\psi}$-induced spreading, contraction [i.e., $\left.\bar{D}_{\eta} \gg d\left(x^{2} / 2\right) / d t\right]$ at all radii $\rho$ :

$$
\left|\frac{\partial \dot{\psi}}{\partial \rho}\right| \ll\left|\frac{q^{\prime} \dot{\psi}}{q}\right|, \quad|\dot{\psi}| \ll \bar{D}_{\eta}\left|\frac{q^{\prime} \psi^{\prime}}{q}\right|, \quad \dot{\psi} \text { conditions. }
$$

Physically, these criteria imply that within the plasma the poloidal flux change must be nearly homogeneous and the induced parallel inductive electric field $\langle\delta \mathbf{E} \cdot \mathbf{B}\rangle$ must be smaller than the equilibrium resistive electric field induced by the parallel current in the plasma, $\eta_{\|}^{\text {nc }}\langle\mathbf{J} \cdot \mathbf{B}\rangle$. A later paper will develop a transient paleoclassical transport theory for situations that violate these conditions.

Some final notes are in order when magnetic fluxes (field lines) are diffusing. While in ideal MHD magnetic-field lines can be assigned unique labels (i.e., Clebsch coordinates $\alpha$ and $\beta$ ), this is no longer possible when plasma resistivity effects are added - because, as an initially localized poloidal magnetic flux diffuses radially according to (76) and (86), it assumes a probability distribution [see (77)] centered about a radial position determined by the advecting radial grid velocity, but with a radial spread (variance) of order $\delta_{e} \sqrt{\bar{\nu}_{e} t}$ that increases with time $t$. Thus, it is better to think of $\psi$ (and $\left.\psi_{*}, x\right)$ as the radial coordinate of a particular flux surface or of magnetic field lines on that surface-and realize that they are not invariant labels for field lines as they diffuse radially. Note also that diffusing poloidal flux does not induce "magnetic flutter" (i.e., $\widetilde{\mathbf{B}} \cdot \boldsymbol{\nabla} \psi \neq 0$ ) or ergodicity of the field lines-because advecting and diffusing field lines always lie on flux surfaces and the solenoidal condition $\boldsymbol{\nabla} \cdot \mathbf{B}=0$ is satisfied automatically at all times by the flux function representations of $\mathbf{B}$ in (3) and (61). In most plasma physics studies the fact that magnetic flux is diffusing radially can be neglected-because the flux-surface labels $\left(\rho, \psi, \psi_{t}, \psi_{*}\right.$, or $\left.x\right)$ remain unchanged. However, diffusion of poloidal magnetic flux is important in driven-dissipative $\left(S_{\psi}\right.$ $\left.=\eta_{\|}^{\mathrm{nc}}\langle\mathbf{J} \cdot \mathbf{B}\rangle / I\left\langle R^{-2}\right\rangle\right)$, current-carrying resistive toroidal plasmas through its effects, on the resistive time scale $\tau_{\eta}$, on the radial position of the guiding center of an electron.

\section{PALEOCLASSICAL KINETICS, ANALYSIS}

Paleoclassical radial transport will be caused by electrons and their heat being nearly "frozen to" and hence carried with the poloidal magnetic flux $\psi$ as it is advected and diffused radially. The Lorentz force $q_{e} \mathbf{v} \times \mathbf{B}$ causes electrons to gyrate rapidly (at the electron gyrofrequency $\omega_{c e}$ $\left.\equiv q_{e} B / m_{e}\right)$ with typical electron gyroradii $\varrho_{e} \equiv v_{T_{e}} / \omega_{c e}$ $\lesssim 0.1 \mathrm{~mm} \ll \delta_{e} \sim 1 \mathrm{~mm}$ around a magnetic-field line. In the absence of magnetic-flux diffusion, a key constant of the motion for axisymmetric systems is the canonical toroidal angular momentum $p_{\zeta} \equiv R^{2} \boldsymbol{\nabla} \zeta \cdot\left(m_{e} \mathbf{v}+q_{e} \mathbf{A}\right)=m_{e} R^{2} \nabla \zeta \cdot \mathbf{v}$ $-q_{e} \psi$ in which $\mathbf{A}=\psi_{t} \boldsymbol{\nabla} \theta-\psi \boldsymbol{\nabla} \zeta$ from (3) has been used. Since to lowest order in a small gyroradius expansion $p_{\zeta} \simeq-q_{e} \psi$, one usually thinks of the electron guiding centers as being "stuck to" the poloidal flux, or at least to the small amount of poloidal magnetic flux $\delta \psi$ traversed by their radial gyromotion. The lowest-order, collisionless distribution function $f$ in standard drift-kinetic and neoclassical theories is a function of the constants of the particle motion, including $p_{\zeta}$. Thus, to lowest order in a small gyroradius expansion it is a function of $\psi: f_{0}=f(\psi)$.

In plasma kinetic theory, and, in particular, drift-kinetic theory, ${ }^{7,22}$ magnetic-flux surfaces and field lines are usually considered to be fixed in space. However, as discussed in Sec. V, when $\eta_{\|}\langle\mathbf{J} \cdot \mathbf{B}\rangle \neq 0$ they diffuse radially. This causes the poloidal flux traversed by electron gyromotion and thus $p_{\zeta} \simeq-q_{e} \psi$, and hence the electron guiding center's $\psi$ position, to become a stochastic (diffusing) variable. To see this, 
consider Newton's second law for electrons with a Lorentz force using $\mathbf{E}=-\partial \mathbf{A} / \partial t$ (i.e., neglecting a potential $\phi$ ):

$$
m_{e} d \mathbf{v} / d t=q_{e}(-\partial \mathbf{A} / \partial t+\mathbf{v} \times \mathbf{B}) .
$$

Taking the projection of this equation in the $\mathbf{e}_{\zeta}=R^{2} \nabla \zeta$ (covariant base vector) direction, one obtains

$$
m_{e} \frac{d\left(R^{2} \boldsymbol{\nabla} \zeta \cdot \mathbf{v}\right)}{d t}=q_{e}\left(\frac{\partial \psi}{\partial t}+\mathbf{v} \cdot \boldsymbol{\nabla} \psi\right)=q_{e} \frac{d \psi}{d t},
$$

which is valid on the gyromotion and longer time scales. (The same equation is applicable for the local helical geometry since the covariant base vector $\mathbf{e}_{\alpha}=R^{2} \nabla \zeta=\mathbf{e}_{\zeta}$.)

Usually one simply integrates this equation over time to obtain the $p_{\zeta}=m_{e} R^{2} \nabla \zeta \cdot \mathbf{v}-q_{e} \psi$ constant of electron motion. However, Sec. V showed that in a current-carrying toroidal plasma the poloidal magnetic flux $\psi$ obeys a diffusion equation-from (65) $d \psi / d t=D_{\eta} \Delta^{+} \psi-S_{\psi}$ for $x^{2}>\bar{\delta}_{e}^{2}$ - and hence (89) cannot be simply integrated over $t$. Tokamaks are driven-dissipative systems in which the flux and energy in the poloidal magnetic field are being continuously injected (via $S_{\psi}$ ) and diffused, dissipated (via $D_{\eta}$ ). Thus, the poloidal magnetic field in tokamaks is a nonconservative field, for which Newton's second law cannot be transformed into an area-preserving set of Hamiltonian equations-at least not on the poloidal field dissipation time scale $\left(\tau_{\eta} \sim a^{2} / 6 D_{\eta}\right)$, i.e., not on the transport time scale.

The effects of advection and diffusion of poloidal flux in driven-dissipative toroidal plasmas with $\eta_{\|}\langle\mathbf{J} \cdot \mathbf{B}\rangle \neq 0$ will be considered via a multiple-time-scale analysis. First, one notes that on the gyromotion time scale $p_{\zeta}$ is a constant of the motion and the radial $\left(\psi_{t}, \rho\right.$ or $\left.x \equiv \rho-\rho_{*}\right)$ position of an electron is oscillatory. Thus, to first order in a small gyroradius expansion one writes

$$
\psi(x, t)=\psi\left(x_{0}\right)+\delta \psi \sin \left(-\omega_{c e} t+\varphi_{0}\right),
$$

in which $x_{0}$ is the radial position of the electron's guiding center and $\delta \psi \sim \varrho_{e} \partial \psi / \partial \rho \ll \psi_{0}$ is the small amount of poloidal magnetic flux traversed by the radial component of electron gyromotion.

Normally one thinks of $\delta \psi$ as an invariant quantity determined by the electron gyroradius. However, when $\eta_{\|}\langle\mathbf{J} \cdot \mathbf{B}\rangle \neq 0$, the small, radially localized flux $\delta \psi$ is advected and diffused radially - as indicated by the Fokker-Planck coefficients given in (83). This effect is small and accumulates to become significant only on a much longer time scale than the electron gyroperiod $\tau \equiv 2 \pi / \omega_{c e}$; it will be taken into account as a net small effect (mapping) per electron gyroperiod. Since the radial $(x)$ excursion of the electron is determined by $\delta \psi(x, t)$, over one electron gyroperiod the gyromotion-traversed flux $\delta \psi$ and hence the electron's guiding center radial position $x_{g}$ is advected and diffused radially by

$$
\left\langle\Delta x_{g}\right\rangle=\left(\bar{u}_{g}+\bar{u}_{\dot{\psi}}\right) \tau, \quad\left\langle\left(\Delta x_{g}\right)^{2}\right\rangle=2 \bar{D}_{\eta} \tau .
$$

This amount of radial diffusion per gyroperiod $\tau$ is quite small: $a^{2}\left\langle\left(\Delta x_{g}\right)^{2}\right\rangle / \varrho_{e}^{2} \sim 4 \pi\left(\bar{\nu}_{e} / \omega_{c e}\right)\left(\delta_{e}^{2} / \varrho_{e}^{2}\right) \sim 10^{-3}$ for a typical case with $\bar{\nu}_{e} \sim 10^{5} / \mathrm{s}$ and $\omega_{c e} \sim 10^{11} / \mathrm{s}$. However, lest one thinks it is negligibly small, note that on the same time-scale
Coulomb collisions induce velocity-space diffusion and hence diffusion of the electron gyroradius, which is the physical origin of classical transport, of only about $\left\langle\left(\Delta \varrho_{e}\right)^{2}\right\rangle / \varrho_{e}^{2} \simeq|\langle\Delta \mathbf{v} \Delta \mathbf{v}\rangle| / v^{2} \sim \nu_{e} \tau \sim\left(\omega_{p} / \omega_{c e}\right)\left(\ln n \lambda_{D}^{3}\right) / n \lambda_{D}^{3}$ $\sim 10^{-6}$ for a typical case with $\omega_{p} \sim \omega_{c e}$ and $n \lambda_{D}^{3} \sim 10^{7}$.

Taking the electron gyromotion period $\tau$ to be the relevant stochastic time step $\Delta t$, the net result of the preceding analysis is that electron guiding centers are advected and diffused radially with the same Fokker-Planck coefficients as those for poloidal magnetic flux (field lines), i.e., those given in (83). These effects will be included in a gyro-averaged kinetic equation by adding a spatial Fokker-Planck operator that represents electron guiding center motion due to magnetic-flux advection and diffusion effects-analogous to how the velocity-space diffusion effects of Coulomb collisions were originally added ${ }^{23}$ to the collisionless Vlasov equation. Because some readers may not regard the preceding intuitive arguments and phenomenological derivation of the Fokker-Planck coefficients for electron guiding center advection and diffusion to be formally proven from first principles, this paper will take them to be the key hypothesis of the paleoclassical model (for current-carrying resistive toroidal plasmas) and proceed assuming they are valid.

The appropriate electron kinetic equation is the gyroaveraged one, which is called the drift-kinetic equation ${ }^{22}$ (DKE). Adding a spatial Fokker-Planck operator $\mathcal{D}\{f\}$ (Refs. 19 and 20) that represents electron guiding center motion induced by magnetic-flux advection and diffusion, the magnetic-field-diffusion-modified drift-kinetic equation (MDKE) becomes

$$
\frac{\partial f}{\partial t}+\mathbf{v}_{g} \cdot \nabla f+\dot{\varepsilon} \frac{\partial f}{\partial \varepsilon}=\mathcal{C}\{f\}+\mathcal{D}\{f\} .
$$

Here, $f=f\left(\mathbf{x}_{g}, \varepsilon, \mu, t\right)$ is the guiding center distribution function, $\mathbf{x}_{g}$ is the guiding center position $(\simeq \mathbf{x}$ because the electron gyroradius is negligibly small), the magnetic moment $\mu \equiv m v_{\perp}^{2} / 2 B$, and kinetic energy $\varepsilon \equiv m v^{2} / 2=m v_{\|}^{2} / 2+\mu B$. Further, the guiding center velocity $\mathbf{v}_{g} \equiv v_{\|} \mathbf{B} / B+\mathbf{v}_{D}$ in which $v_{\|} \equiv \mathbf{B} \cdot \mathbf{v} /|\mathbf{B}|= \pm \sqrt{\left(2 / m_{e}\right)(\varepsilon-\mu B)}$ is the parallel (to $\mathbf{B}$ ) component of the electron velocity $\mathbf{v}$ and $\mathbf{v}_{D}$ is the electron drift velocity, $\dot{\varepsilon}=\mu \partial B / \partial t+q_{e} \mathbf{v}_{g} \cdot \mathbf{E} \simeq q_{e} u_{\|} \mathbf{E} \cdot \mathbf{B} / B$, and $\mathcal{C}\{f\}$ is the gyromotion-averaged Coulomb collision operator.

Finally, effects due to magnetic-flux (field line) advection and diffusion on the positions of electron guiding centers are indicated by the Fokker-Planck spatial diffusion operator $(\mathcal{D})$. Because of the complexity of the magnetic geometry, the Fokker-Planck coefficients must be written in a general vectorial form, which is in terms of the covariant base vector in the "radial" direction $\mathbf{e}_{\rho} \equiv \partial \mathbf{x} / \partial \rho=\sqrt{g} \boldsymbol{\nabla} \theta$ $\times \nabla \zeta$, for which $\mathbf{e}_{\rho} \cdot \nabla \rho=1$ :

$$
\frac{\langle\Delta \mathbf{x}\rangle}{\Delta t} \equiv \frac{\left\langle\Delta x_{g}\right\rangle}{\tau} \mathbf{e}_{\rho}, \quad \frac{\langle\Delta \mathbf{x} \Delta \mathbf{x}\rangle}{\Delta t} \equiv \frac{\left\langle\left(\Delta x_{g}\right)^{2}\right\rangle}{\tau} \mathbf{e}_{\rho} \mathbf{e}_{\rho} .
$$

In the paleoclassical model electron guiding centers are advected and diffused with the poloidal field lines. Thus, the Fokker-Planck coefficients $\left\langle\Delta x_{g}\right\rangle / \tau$ and $\left\langle\left(\Delta x_{g}\right)^{2}\right\rangle / \tau$ are as specified in (91). In full three-dimensional geometry the Fokker-Planck differential operator is ${ }^{19,20}$ 


$$
\mathcal{D}\{f\} \equiv-\boldsymbol{\nabla} \cdot \frac{\langle\Delta \mathbf{x}\rangle}{\Delta t} f+\frac{1}{2} \boldsymbol{\nabla} \cdot\left(\boldsymbol{\nabla} \cdot \frac{\langle\Delta \mathbf{x} \Delta \mathbf{x}\rangle}{\Delta t} f\right) .
$$

Using (12), the definition of the divergence of a vector field, $\boldsymbol{\nabla} \cdot \mathbf{A} \equiv \Sigma_{i}(1 / \sqrt{g})\left(\partial / \partial u^{i}\right)\left(\sqrt{g} \mathbf{A} \cdot \boldsymbol{\nabla} u^{i}\right), \quad$ and neglecting $\left\langle\nabla \rho \cdot \partial \mathbf{e}_{\rho} / \partial \rho\right\rangle=\left\langle\nabla \rho \cdot \partial^{2} \mathbf{x} / \partial \rho^{2}\right\rangle \sim \mathcal{O}\left(\epsilon^{2}\right)$, when the distribution function $f$ is solely a function of a magnetic-flux coordinate (i.e., $x, \rho, \psi$ or $\psi_{*}$ ), the flux-surface average of this operator becomes

$$
\begin{aligned}
\langle\mathcal{D}\{f(\rho)\}\rangle & \simeq \frac{1}{V^{\prime}} \frac{\partial}{\partial \rho}\left[-V^{\prime} \frac{\left\langle\Delta x_{g}\right\rangle}{\tau} f+\frac{\partial}{\partial \rho} V^{\prime} \frac{\left\langle\left(\Delta x_{g}\right)^{2}\right\rangle}{2 \tau} f\right] \\
& =\frac{1}{V^{\prime}} \frac{\partial}{\partial \rho}\left[-V^{\prime}\left(\bar{u}_{g}+\bar{u}_{\dot{\psi}}\right) f+\frac{\partial}{\partial \rho} V^{\prime} \bar{D}_{\eta} f\right] .
\end{aligned}
$$

\section{A. Axisymmetric paleoclassical transport}

Transport induced by adding electron guiding center diffusion effects to the DKE will be explored first for a toroidally axisymmetric distribution function (i.e., $\partial f / \partial \zeta=0$ ). Then, using the usual magnetic-field model in (3), (92) can be simplified to

$$
\frac{\partial f}{\partial t}+\left.\frac{\boldsymbol{v}_{\|}}{B}(\mathbf{B} \cdot \boldsymbol{\nabla} \theta) \frac{\partial f}{\partial \theta}\right|_{\psi}+\mathbf{v}_{D} \cdot \boldsymbol{\nabla} f+\dot{\varepsilon} \frac{\partial f}{\partial \varepsilon}=\mathcal{C}\{f\}+\mathcal{D}\{f\} .
$$

Here, $f=f_{a}(\psi, \theta, \mu, \varepsilon, t)$, in which the subscript $a$ indicates the axisymmetric distribution, and from (6) and (7) $\mathbf{B} \cdot \boldsymbol{\nabla} \theta$ $=\psi^{\prime} / \sqrt{g} \simeq B_{p} / r=B_{t} / R_{0} q$.

As usual, ${ }^{7,8}$ solutions of the kinetic equation will be sought using a small gyroradius ordering scheme in which the first-order terms are linear in the gyroradius, em skin depth $\delta_{e}$, and parallel electric field $E_{\|}$. The second-order terms are quadratic in these quantities and cause transporttime-scale temporal evolution effects.

The lowest (zeroth)-order axisymmetric DKE obtained from (96), which includes parallel streaming $\left[u_{\|}(\mathbf{B} \cdot \nabla \theta) / B\right.$ $\sim v_{T_{e}} / R_{0} q \sim \omega_{t}$, transit frequency] and Coulomb collision frequency $\left(\sim \nu_{e}\right)$ effects on the lowest-order axisymmetric distribution function $f_{a 0}$, is

$$
\left.\frac{\underline{u}_{1}}{B}(\mathbf{B} \cdot \boldsymbol{\nabla} \theta) \frac{\partial f_{a 0}}{\partial \theta}\right|_{\psi}=\mathcal{C}\left\{f_{a 0}\right\} .
$$

Its solution (for low-collisionality plasmas in which $\lambda_{e}$ $\equiv v_{T_{e}} / \nu_{e}>\pi \bar{R} q$, the poloidal periodicity length) is ${ }^{7}$

$$
f_{a 0}=f_{M}(\psi, \varepsilon, t)=n_{e}(\psi, t)\left[\frac{m_{e}}{2 \pi T_{e}(\psi, t)}\right]^{3 / 2} e^{-\varepsilon / T_{e}(\psi, t)} .
$$

Thus, the lowest-order axisymmetric distribution is a Maxwellian that is a function of the poloidal flux function $\psi$; hence, the electron temperature and density are constant on the (slowly evolving) $\psi(\rho, t)$ flux surfaces. The parametric dependence of the electron density $n_{e}$ and temperature $T_{e}$ on the time $t$ allows for temporal evolution of these quantities on the transport time scale, which is second order in the small gyroradius expansion.

The first-order drift-kinetic equation is ${ }^{7}$

$$
\frac{v_{\|}}{B}(\mathbf{B} \cdot \boldsymbol{\nabla} \theta) \frac{\partial f_{1}}{\partial \theta}-\mathcal{C}\left\{f_{1}\right\}=-\mathbf{v}_{D} \cdot \nabla \psi \frac{\partial f_{M}}{\partial \psi}-\frac{e u_{\|} E_{\|}}{T_{e}} f_{M} .
$$

Solutions of it yield ${ }^{7,8}$ the first order (in the small gyroradius expansion): current induced by the inductive parallel electric field [which leads to the Spitzer electrical resistivity (53)], neoclassical flows and heat flows within the flux surface induced by $\mathbf{v}_{D} \cdot \nabla f_{M}$, and the closure relations for the parallel viscous stress in (42) and (45).

The net electron energy transport equation including both neoclassical and axisymmetric paleoclassical effects is of second order. It is obtained by taking the kinetic-energy moment $\left[\int d^{3} v\left(m_{e} v^{2} / 2\right)\right]$ of the axisymmetric MDKE (96), flux-surface averaging it using $(10),{ }^{7}$ and approximating $\mathcal{D}\left\{f_{a}\right\} \simeq \mathcal{D}\left\{f_{M}(\psi)\right\}$ to yield

$$
\begin{gathered}
\frac{3 / 2}{V^{\prime 5 / 3}} \frac{\partial}{\partial t}\left(n_{e} T_{e} V^{\prime 5 / 3}\right)+\frac{\partial}{\partial V}\left\langle\left(\mathbf{q}_{e}^{\mathrm{nc}}+\frac{5}{2} T_{e} \boldsymbol{\Gamma}_{e}^{\mathrm{nc}}\right) \cdot \boldsymbol{\nabla} V\right\rangle \\
+\frac{\partial}{\partial V}\left\langle\mathbf{Q}_{e a}^{\mathrm{pc}} \cdot \boldsymbol{\nabla V} V\right\rangle=Q_{e} .
\end{gathered}
$$

Here, since the collisional entropy is $s_{e} \equiv(3 / 2) \ln p_{e} V^{5 / 3}$, the first term represents $p_{e} \partial s_{e} / \partial t$-adiabatic compression changes ${ }^{7}$ in electron pressure $p_{e} \equiv n_{e} T_{e}$ on a flux surface $\left[V \rightarrow V^{\prime}(\psi)\right]$ in the presence of moving poloidal flux surfaces $(\dot{\psi} \neq 0)$. The other terms represent entropy-producing processes: neoclassical conductive $\left(\mathbf{q}_{e}^{\text {nc }}\right)$ and convective $\left[(5 / 2) T_{e} \Gamma_{e}^{\mathrm{nc}}\right]$ electron heat fluxes, the total (advective plus diffusive) axisymmetric paleoclassical electron heat flux $\left(\mathbf{Q}_{e a}^{\mathrm{pc}}\right)$, and electron heating $\left(Q_{e}\right)$ due to collisional effects (joule heating, electron viscosity, and collisions with ions). The neoclassical conductive heat flux (in the $\mathbf{u}_{g}$ rest frame) is in a Fourier heat flux form $\left(\mathbf{q}_{e} \equiv-n_{e} \chi_{e}^{\chi^{\mathrm{nc}}} \boldsymbol{\nabla} T_{e}\right)$ and hence diffusive: $\quad(\partial / \partial V)\left\langle\mathbf{q}_{e}^{\mathrm{nc}} \cdot \boldsymbol{\nabla} V\right\rangle \simeq-\left(1 / V^{\prime}\right)(\partial / \partial \rho)$ $\times\left(V^{\prime}\left\langle|\nabla \rho|^{2}\right\rangle n_{e} \chi_{e}^{\mathrm{nc}} \partial T_{e} / \partial \rho\right)$ in which $\chi_{e}^{\mathrm{nc}} \sim \mu_{e} \varrho_{p e}^{2}$ $\sim \nu_{e} \varrho_{e}^{2} q^{2} / \epsilon^{3 / 2}$ is the neoclassical electron heat diffusivity. ${ }^{7,8}$

The axisymmetric paleoclassical electron heat transport $\left\langle\boldsymbol{\nabla} \cdot \mathbf{Q}_{e a}^{\mathrm{pc}}\right\rangle=(\partial / \partial V)\left\langle\mathbf{Q}_{e a}^{\mathrm{pc}} \cdot \boldsymbol{\nabla} V\right\rangle$ is obtained from the kineticenergy moment of $\mathcal{D}\left\{f_{M}\right\}$ :

$$
\begin{aligned}
\frac{\partial}{\partial V}\left\langle\mathbf{Q}_{e a}^{\mathrm{pc}} \cdot \boldsymbol{\nabla} V\right\rangle \equiv & -\int d^{3} v \frac{m_{e} v^{2}}{2}\left\langle\mathcal{D}\left\{f_{M}(\psi)\right\}\right\rangle \\
= & \frac{1}{V^{\prime}} \frac{\partial}{\partial \rho}\left[V^{\prime}\left(\bar{u}_{g}+\bar{u}_{\psi}\right) \frac{3}{2} n_{e} T_{e}\right. \\
& \left.-\frac{\partial}{\partial \rho}\left(V^{\prime} \bar{D}_{\eta^{2}} \frac{3}{2} n_{e} T_{e}\right)\right] .
\end{aligned}
$$

Physically, the electron internal energy $(3 / 2) n_{e} T_{e}$ is advected and compressed by the grid velocity $u_{g}$ and the poloidal field-line motion induced by $\dot{\psi} \neq 0$, and diffused radially because of the magnetic-field diffusivity $D_{\eta}$. When the advective flow is measured in the rest frame of the toroidal flux surfaces (i.e., $\mathbf{u}_{g}$ ), as neoclassical flows are, the $\bar{u}_{g}$ contribution to (101) is removed.

The diffusive paleoclassical part of the electron heat transport in (101) indicates an axisymmetric (subscript $a$ ) paleoclassical radial electron heat diffusivity of 


$$
\chi_{e a}^{\mathrm{pc}}=\frac{3}{2} D_{\eta}=\frac{3}{2} \frac{\eta_{\|}^{\mathrm{nc}}}{\mu_{0}}=\frac{3}{2} \bar{\nu}_{e} \delta_{e}^{2}, \quad \text { axisymmetric } \chi_{e}^{\mathrm{pc}} .
$$

Note that this paleoclassical electron heat diffusivity is usually larger than the neoclassical electron heat diffusivity $\left[\chi_{e a}^{\mathrm{pc}} / \chi_{e}^{\mathrm{nc}} \sim\left(\bar{\nu}_{e} \delta_{e}^{2}\right) /\left(\nu_{e} \varrho_{e}^{2} q^{2} / \epsilon^{3 / 2}\right) \sim \epsilon^{3 / 2} / \beta_{e} q^{2}>1\right], \quad$ especially near the plasma edge where $T_{e}$ and $\beta_{e} \equiv n_{e} T_{e} /\left(B^{2} / 2 \mu_{0}\right)$ are small. Thus, even the axisymmetric paleoclassical effects due to diffusing poloidal flux (field lines) are important for collisional radial electron heat transport in resistive currentcarrying toroidal plasmas.

\section{B. Representations of nonaxisymmetric $f$}

Next, consider the Fourier expansion of the distribution function in the poloidal $(\theta)$ and toroidal $(\zeta)$ angles:

$$
\begin{aligned}
f(\psi, \theta, \zeta) & =\sum_{m, n} f_{m n}(\psi) e^{i m \theta-i n \zeta} \equiv f_{a}+f_{\mathrm{na}} \\
& =\sum_{m} f_{m 0} e^{i m \theta}+\sum_{m, n \neq 0} f_{m n} e^{i m \theta-i n \zeta} .
\end{aligned}
$$

The $n=0$ contributions (first sum on the second line) represent the axisymmetric distribution function $f_{a}$ that has been calculated in Sec. VI A. The nonaxisymmetric $(n \neq 0$, subscript "na") contributions will be relevant along field lines on the "fast" parallel $T_{e}$ equilibration time scale (i.e., $\left.t \sim 1 / \nu_{e}\right)$. On and in the vicinity of a rational surface where $q\left(\rho_{*}\right)=q_{*}=m / n$, the appropriate angular variables are $\theta$ and the helical angle $\alpha \equiv \zeta-q_{*} \theta=\zeta-(m / n) \theta$. The $f_{\text {na }}$ can be put into a form that isolates the helical and poloidal angle dependences by first changing the poloidal harmonic sum in (103) from $m$ to $m^{\prime}$, next making use of the definition of the helical angle $\alpha$, and finally defining a new poloidal harmonic summation variable $\tilde{m} \equiv m^{\prime}-m$ :

$$
f_{\mathrm{na}}(\psi, \theta, \alpha)=\sum_{n \neq 0} e^{-i n \alpha} \sum_{\tilde{m}} f_{m+\widetilde{m}, n}(\psi) e^{i \tilde{m} \theta} .
$$

Since $\alpha \equiv \zeta-(m / n) \theta, \Sigma_{n \neq 0}$ implicitly involves a sum over $m$ for $n \min \{q\} \leqslant m \leqslant n \max \{q\}$ for each $n$.

In Sec. VI C $f_{\text {na }}$ "helical" kinetics and paleoclassical radial electron heat transport in the vicinity of a medium-order $(n \sim 10)$ rational surface will be considered. The basic philosophy will be that on and near a rational field line there is helical symmetry (or "quasihelical symmetry") in the toroidal system, and that in a low-collisionality plasma the electron temperature will equilibrate over the long parallel length of helical field lines (if $\lambda_{e}>\ell_{*}$ ). Local helical flux coordinates (in particular, the local radial variable $x \equiv \rho-\rho_{*}$ and helical angle $\left.\alpha \equiv \zeta-q_{*} \theta\right)$ will be relevant because the electron temperature will be equilibrated along the long parallel extent of a helical field line as it diffuses radially away from the rational surface-into regions with $q \neq q_{*}$, because of shear (i.e., $q^{\prime} \neq 0$ ) in the magnetic field.

Developing a useful (i.e., one-dimensional) representation of $f_{\text {na }}$ for $n \gg 1$ is analogous to the development of ballooning mode theory. ${ }^{24,25}$ The basic issue is how does one maintain periodicity of solutions in the poloidal $(\theta)$ and helical $(\alpha)$ angles as one moves radially away from a helically symmetric rational surface (i.e., to $x \neq 0$ in a sheared mag- netic field). The procedure for doing this for large $n$ "flutelike" plasma responses that are extended long distances $(|\ell| \gg \pi \bar{R} q)$ along field lines, such as for paleoclassical processes, is to assume $q$ is locally approximately a linear function of $x$ (i.e, $q \simeq q_{*}+x q^{\prime}$ ) and use a ballooning mode representation. ${ }^{24,25}$ Subsequent paragraphs in this section develop a useful form for $f_{\text {na }}$ following the procedure Lee and Van $\operatorname{Dam}^{25}$ used to develop a ballooning representation.

Neglecting the parallel electric field $E_{\|}$and hence the $\dot{\varepsilon} \partial f / \partial \varepsilon$ term, whose dominant effects (for $n \gg 1$ ) have already been included in axisymmetric neoclassical theory, the relevant nonaxisymmetric MDKE (92) is

$$
\partial f / \partial t+\left(\boldsymbol{u}_{\|} / B\right) \mathbf{B} \cdot \boldsymbol{\nabla} f+\mathbf{v}_{D} \cdot \boldsymbol{\nabla} f=\mathcal{C}\{f\}+\mathcal{D}\{f\} .
$$

In the vicinity of a given rational surface the magnetic field can be represented in terms of its helical and magnetic-shear components as in (61) and the parallel-streaming differential operator in (105) becomes: ${ }^{18}$

$$
\mathbf{B} \cdot \boldsymbol{\nabla} f=(\mathbf{B} \cdot \boldsymbol{\nabla} \theta)\left[\left.\frac{\partial f}{\partial \theta}\right|_{\psi_{*}, \alpha}+\left.\left(q-q_{*}\right) \frac{\partial f}{\partial \alpha}\right|_{\psi_{*}, \theta}\right] .
$$

Thus, on and in the vicinity of the $q_{*} \equiv m / n$ rational surface the relevant distribution function becomes $f \rightarrow f\left(\psi_{*}, \theta, \alpha, \mu, \varepsilon, t\right)$. As before, from the definition of the Jacobian in (7), B $\cdot \nabla \theta=\psi^{\prime} / \sqrt{g} \simeq B_{p} / r=B_{t} / R_{0} q$.

Applying this parallel-streaming operator to the nonaxisymmetric distribution form given in (104) yields

$$
\begin{aligned}
\mathbf{B} \cdot \boldsymbol{\nabla} f_{\mathrm{na}}= & (\mathbf{B} \cdot \boldsymbol{\nabla} \theta) \sum_{n \neq 0} e^{-i n \alpha} \sum_{\tilde{m}} e^{i \tilde{m} \theta} i\left[\tilde{m}-n\left(q-q_{*}\right)\right] \\
& \times f_{m+\tilde{m}, n}(\psi) .
\end{aligned}
$$

Since the parallel-streaming term $\left(\boldsymbol{v}_{\|} / B\right) \mathbf{B} \cdot \boldsymbol{\nabla} f_{\text {na }}$ is dominant in (105), it will cause the Fourier coefficient $f_{m+\tilde{m}, n}$ to be quite small (i.e., negligible) unless its multiplier $\tilde{m}-n\left(q-q_{*}\right)$ is small. Near the $q_{*} \equiv m / n$ rational surface $q$ can be expanded in a Taylor series as given in (18) and the free-streaming coefficient becomes

$$
\tilde{m}-n\left(q-q_{*}\right) \simeq \tilde{m}-n\left(m / n+x q^{\prime}-q_{*}\right)=\tilde{m}-n x q^{\prime} .
$$

This will be smallest and lead to the largest $f_{m+\tilde{m}, n}$ for $\tilde{m}=0$ and $\left|n x q^{\prime}\right| \ll 1$. The resulting "helically resonant" Fourier coefficient (near $q=q_{*}$ ) will be

$$
f_{*}(x) \equiv f_{m, n}\left(\psi_{*}\right), \quad \text { helical kinetic distribution. }
$$

Here, the $*$ and $m, n$ subscripts indicate the helical Fourier coefficients for the rational surface $q_{*} \equiv m / n$ and the argument has been changed from the poloidal $(\psi)$ to helical $\left(\psi_{*} \propto x^{2}\right)$ flux, which is the appropriate (radial) flux label near the given rational surface. Because from (22) the spacing between rational surfaces with different $m$ but the same $n$ is $\Delta \simeq 1 / n q^{\prime}$, the criterion $\left|n x q^{\prime}\right| \ll 1$ is just $|x| \ll \Delta$. Hence, the $f_{*}(x)$ solutions will be highly peaked near the $q_{*}$ rational surface; in particular, they will be radially localized to a region much narrower than the spacing between same $n$ rational surfaces. 
Since the coefficients in (105) do not vary much with $\theta$ (and hence $m$ in a Fourier expansion), except for the drift effects (due to $\mathbf{v}_{D}$ ) which are higher order and hence small, another important property of the $f_{m+\tilde{m}, n}$ Fourier coefficients is that they will be (at least approximately) translationally invariant:

$$
f_{m+\widetilde{m}, n}(x)=e^{i \lambda} f_{m+\widetilde{m}-1, n}(x-\Delta)=\cdots=e^{i \tilde{m} \lambda} f_{*}(x-\tilde{m} \Delta)
$$

in which $\lambda \sim \pi / n q \sim 1 / n \ll 1$ is a small phase factor that represents a radial envelope. ${ }^{25}$ Using this result and $\Delta \equiv 1 / n q^{\prime}$ in the representation of the nonaxisymmetric $f_{\text {na }}$ in (104) yields

$$
f_{\mathrm{na}} \simeq \sum_{n \neq 0} e^{-i n \alpha} \sum_{\tilde{m}=-\infty}^{\infty} f_{*}\left(\widetilde{m}-n x q^{\prime}\right) e^{i \widetilde{m}(\theta+\lambda)} .
$$

This is transformed using the Poisson sum formula ${ }^{26}$

$$
\sum_{\tilde{m}=-\infty}^{\infty} F(\widetilde{m})=\sum_{p=-\infty}^{\infty} \hat{F}(2 \pi p)
$$

in which $\hat{F}(p)$ is the Fourier transform of $F(\widetilde{m})$. The Fourier transform of the Fourier coefficient in (111) is

$$
\begin{aligned}
\hat{F}(p) & \equiv \int_{-\infty}^{\infty} d \tilde{m} e^{i p \tilde{m}}\left[f_{*}\left(\tilde{m}-n x q^{\prime}\right) e^{i \widetilde{m}(\theta+\lambda)}\right] \\
& =\int_{-\infty}^{\infty} d z e^{i\left(z+n x q^{\prime}\right)(\theta+\lambda+p)} f_{*}(z) \\
& =e^{i n x q^{\prime}(\theta+\lambda+p)} \hat{f}_{*}(\theta+\lambda+p) .
\end{aligned}
$$

Using this result together with the Poisson sum formula (112) in (111) yields the representation

$$
f_{\text {na }} \simeq \sum_{n \neq 0} e^{-i n \alpha} \sum_{p=-\infty}^{\infty} \hat{f}_{*}(\theta+\lambda+2 \pi p) e^{i n x q^{\prime}(\theta+\lambda+2 \pi p)} .
$$

Note that this is a periodic function of both the poloidal angle $\theta$ and the helical angle $\alpha$. Since near a rational surface where $q \simeq q_{*}+x q^{\prime}$ the Clebsch coordinate $\beta \equiv q \theta-\zeta$ is approximately $-\alpha+x q^{\prime} \theta$, this form represents a locally periodic Clebsch magnetic-field representation $\left(\sim e^{i n \beta}\right)$. Also, $\theta \rightarrow \theta$ $+\lambda+2 \pi p$ represents extending the poloidal angle $\theta$ into a field-line variable along $\mathbf{B}$. [At a minimum in $q$ one has $q^{\prime}=0$; in the vicinity of a $q_{\min }$ rational surface the Fourier modes in (114) are replaced by "modelets" ${ }^{, 27,28}$ and the ballooning representation theory proceeds qualitatively in the same manner.]

To put this "ballooning representation" in a more intuitive form one recognizes that (for $n \gg 1$ ) the distance $\ell$ along a helical magnetic-field line is $(-\pi \leqslant \theta \leqslant \pi)$,

$$
\ell \simeq(\theta+\lambda+2 \pi p) \bar{R} q_{*}, \quad \text { distance along } \mathbf{B} .
$$

Using $\Delta p=\Delta \ell / 2 \pi \bar{R} q_{*}$ to convert the discrete sum over $p$ in (114) into a continuous integral over $\ell$, one obtains

$$
f_{\mathrm{na}} \simeq \sum_{n \neq 0} e^{-i n \alpha} \int_{-\ell_{*}}^{\ell_{*}} \frac{d \ell}{2 \pi \bar{R} q_{*}} e^{i k_{\|}(x) \ell} \hat{f}_{*}(\ell) .
$$

Here, for the sheared $\left(q^{\prime} \neq 0\right)$ magnetic field,

$$
k_{\|}(x) \equiv \frac{n x q^{\prime}}{\bar{R} q_{*}}, \quad \text { effective parallel wave number. }
$$

Taking the flux-surface average of (111) and comparing the result to the $f_{\text {na }}$ representation in (116), one sees that [neglecting $\mathcal{O}\left(\epsilon^{2}\right)$ corrections]

$$
f_{*}(x) \simeq \int_{-\ell_{*}}^{\ell_{*}} \frac{d \ell}{2 \pi \bar{R} q_{*}} e^{i k_{\|}(x) \ell} \hat{f}_{*}(\ell) .
$$

The limits on the $\ell$ integration in (116) and (118) are given by the half-length of a helical field line $\left[\ell_{*} \equiv \pi \bar{R} q_{*} n\right.$ from (16)] because finite $n$ helical field lines have a finite length; thus, the limits take account of the fact that the distribution function $\hat{f}_{*}(\ell)$ should be periodic over the length $2 \ell_{*}=2 \pi \bar{R} q_{* n}$. These limits also imply that the sum over $p$ in (114) only extends from $-n / 2$ to $n / 2$ - to represent summing over $n$ poloidal transits of a helical field line in the vicinity of a $q_{*}=m / n$ rational flux surface. Since $\hat{f}_{*}(\ell)$ is usually nearly constant (see Sec. VID) for $|\ell| \leqslant \ell_{*}$, the $\ell$ integration in (116) and (118) will yield factors of approximately $\ell_{*} / \pi \bar{R} q_{*}=n \gg 1$, which will produce the multiplier $M$ [see (134) and (137) below] in the paleoclassical electron heat diffusivity-physically because contributions of $n$ poloidal passes of the helical field line must be summed to obtain the net response for one poloidal period of the plasma, as is done in ballooning mode theory. ${ }^{24,25}$ Note that the present analysis is only valid asymptotically since an $n \gg 1$ approximation has been used throughout the derivation.

In the ballooning representation the parallel distance $\ell$ is proportional to the Fourier transform variable $k_{x}$ for the $x$ (radial) variation of $f_{*}(x)$. Also, one has

$$
k_{\|}(x) \ell=k_{x}(\ell) x,
$$

where $k_{x}(\ell) \equiv n q^{\prime}\left(\ell / \bar{R} q_{*}\right)=n q^{\prime}(\theta+\lambda+2 \pi p)$, which is $k_{\theta} s \theta$ in the usual balloooning mode theory ${ }^{24,25}$ with $k_{\theta} \equiv n q / \rho$ and $s \equiv \rho q^{\prime} / q$.

Satisfaction of the criterion $k_{x}^{2}(\ell) \bar{\delta}_{e}^{2}<1$ (or $|x|^{2}>\bar{\delta}_{e}^{2}$ ) for diffusing helical magnetic-field lines-see discussion between (75) and (76) -requires $|\ell|<\ell_{\delta}$ in which

$$
\ell_{\delta} \equiv \bar{R} q_{*} /\left(n \bar{\delta}_{e} q^{\prime}\right), \quad \text { diffusing field-line length. }
$$

This length is equal to or longer than the helical rational field-line length $\ell_{*} \equiv \pi \bar{R} q_{* n}$ (16) for $n \leqslant n_{\max }$ in which

$$
n_{\max } \equiv 1 /\left(\pi \bar{\delta}_{e} q^{\prime}\right)^{1 / 2}, \quad \text { maximum } n \text { for } \ell_{\delta} \geqslant \ell_{*} .
$$

As after (22), if $q^{\prime}$ is negative it is to be replaced by its absolute value $\left|q^{\prime}\right|$. Near a minimum in $q$ where $q^{\prime}$ gets small or vanishes (i.e., near the magnetic axis or a minimum in $q$ for negative central shear discharges), $n_{\max }$ is bounded by 


$$
\max \left\{n_{\max }\right\}=1 /\left(\pi^{2} \bar{\delta}_{e}^{2} q^{\prime \prime}\right)^{1 / 3} \quad \text { near } q^{\prime}=0 .
$$

This limit is obtained from $\ell_{\delta} \geqslant \ell_{*}$ with $q^{\prime}=x q^{\prime \prime}$ $=\left(q^{\prime \prime} / n_{\max }\right)^{1 / 2}$ deduced from $\Delta q=\left(x^{2} / 2\right) q^{\prime \prime}$ using the fact the furthest one can be from a $n_{\max }$ rational surface is $\Delta q=1 /\left(2 n_{\max }\right)$. The length of helical field lines that are diffusing radially over their entire parallel length is

$$
\ell_{\max } \equiv \pi \bar{R} q_{*} n_{\max }, \quad \text { maximum diffusing length. }
$$

\section{Nonaxisymmetric (helically resonant) paleoclassical transport}

Properties of solutions of the nonaxisymmetric MDKE (105) on the electron collision time scale will be sought using an ordering scheme in which the transit frequency $\omega_{t} \sim v_{\mid}(\mathbf{B} \cdot \nabla \theta) / B \sim v_{T e} / R_{0} q$ is larger than all other frequencies. Then, to lowest order the nonaxisymmetric MDKE $(105)$ is $\left(v_{\|} / B\right)(\mathbf{B} \cdot \nabla \theta) \partial f_{\mathrm{na} 0} / \partial \theta=0$, which indicates that to lowest order $f_{\text {na }}$ is independent of the poloidal angle $\theta$. Thus, the representation of $f_{\mathrm{na}}$ in (116) can be used for $f_{\text {na0 }}$ with $\hat{f}_{*_{0}}=\hat{f}_{*_{0}}(\ell, \varepsilon, \mu, \rho, t)$ in which the parametric $\rho, t$ dependences allow for evolution of $\hat{f}_{*_{0}}$ and hence $f_{\text {na }}$ on the transport time scale.

Drift effects are higher order in a small gyroradius expansion and are nelected for now; they will be discussed in the next to last paragraph of this subsection. Thus, for the next order nonaxisymmetric MDKE, using $f=f_{\text {na0 }}$ from (116), the parallel-streaming operator in (106) with $q-q_{*} \simeq x q^{\prime}, \quad$ inx $q^{\prime} f_{*}(x) \rightarrow \bar{R} q_{*} \partial \hat{f}_{*}(\ell) / \partial \ell, \quad$ and $\quad \partial f_{*} / \partial x$ $\rightarrow i\left(\partial k_{\|} / \partial x\right) \ell \hat{f}_{*}=i k_{x}(\ell) \hat{f}_{*}$ in part of $\mathcal{D}\left\{f_{* 0}\right\} \quad$ so $\mathcal{D}\left\{f_{*_{0}}\right\}$ $\simeq \int_{-\ell_{*}}^{\ell_{*}} d \ell /\left(2 \pi \bar{R} q_{*}\right) e^{i k_{\|}(x) \ell}\left(-k_{x}^{2} \bar{D}_{\eta} \hat{f}_{*_{0}}+\mathcal{D}\left\{\hat{f}_{*_{0}}\right\}\right) \quad$ (neglecting small advective effects), after bounce averaging to eliminate an undesired next order $f_{\text {na1 }}$ term, one obtains

$$
\frac{\partial \hat{f}_{*_{0}}}{\partial t}+\bar{v}_{\|} \frac{\partial \hat{f}_{*_{0}}}{\partial \ell}+k_{x}^{2}(\ell) \bar{D}_{\eta} \hat{f}_{*_{0}}=\left\langle\mathcal{C}\left\{\hat{f}_{*_{0}}\right\}\right\rangle_{\theta}+\left\langle\mathcal{D}\left\{\hat{f}_{*_{0}}\right\}\right\rangle_{\theta}
$$

Here, \langle\rangle$_{\theta}$ indicates a bounce average,

$$
\langle A(\theta)\rangle_{\theta} \equiv \frac{\oint B \sqrt{g} d \theta A(\theta) / u_{\|}}{\oint B \sqrt{g} d \theta / u_{\|}}=\frac{\left\langle B A(\theta) / u_{\|}\right\rangle}{\left\langle B / u_{\|}\right\rangle},
$$

and a bounce-averaged parallel speed has been defined: $\overline{v_{\|}} \equiv\langle B\rangle /\left\langle B / v_{\|}\right\rangle$, which results from $\left\langle\left(v_{\|} / B\right) \mathbf{B} \cdot \boldsymbol{\nabla} \theta\right\rangle_{\theta}$ $=\langle B\rangle /\left(\bar{R} q_{*}\left\langle B / u_{\|}\right\rangle\right.$using the relation $4 \pi^{2} \psi^{\prime} / V^{\prime}=\langle B\rangle / \bar{R} q_{*}$ deduced from (17).

To obtain the lowest-order version of (124), one drops the time dependence and magnetic diffusion induced effects $\left[k_{x}^{2}(\ell) \bar{D}_{\eta} \hat{f}_{*_{0}}\right.$ and $\left.\mathcal{D}\left\{\hat{f}_{*_{0}}\right\}\right]$ to obtain simply

$$
\overline{v_{\|}} \partial \hat{f}_{* 0}(\ell) / \partial \ell=\left\langle\mathcal{C}\left\{\hat{f}_{* 0}\right\}\right\rangle_{\theta} .
$$

A solution of this equation is a Maxwellian distribution that is independent of $\ell$ (i.e., distance along a field line):

$$
\hat{f}_{* 0}=f_{M}(\rho, \varepsilon, t)=f_{a 0}=n_{e}(\rho, t)\left[\frac{m_{e}}{2 \pi T_{e}(\rho, t)}\right]^{3 / 2} e^{-\varepsilon / T_{e}(\rho, t)}
$$

Physically, the electron distribution is Maxwellianized and the electron temperature equilibrated along the helical field lines by parallel electron heat conduction. This local helical distribution is fixed to the same normalization as the axisymmetric Maxwellian distribution $f_{a 0}=f_{M}(\psi, \varepsilon, t)$ in (98) because the helical field lines on a $\rho=\rho_{*}$ surface are just the field lines that fill the poloidal flux surface $\psi$ on the $q_{*}$ rational surface. It will be shown below [in (138)] that when this $\hat{f}_{*_{0}}$ is used in (116), the resultant $f_{\text {na }}$ represents the equilibration (for $t \gtrsim 1 / \nu_{e}$ ) of the distribution function along field lines before particle drifts in the $\alpha$ direction cause the distribution to become homogeneous on a flux surface (i.e., cause $\partial f_{\text {na }} / \partial \alpha \rightarrow 0$ and hence $f_{\text {na }} \rightarrow 0$ ). The parametric dependences of the electron density $n_{e}$ and temperature $T_{e}$ on $\rho, t$ allow for temporal evolution of these quantities on longer (i.e., transport) time scales.

It is important to understand the limits of applicability of the Maxwellian distribution solution in (127). They result from considering the effects due to the terms neglected in approximating (124) by (126) and to the neglected drift terms. Temporally, the quasiequilibrium Maxwellian solution in (127) is applicable on time scales longer than the electron collision time $\left(t>1 / \nu_{e}\right)$ but shorter than the (next order) drift time scale $1 / \omega_{D}$ (see next to last paragraph of this subsection).

The parallel length along a helical field line over which (127) applies can be inferred by first considering the effects of the parallel-streaming operator $\bar{v}_{\|} \partial \hat{f}_{*_{0}} / \partial \ell$ in (126). When the electron collision length $\lambda_{e}$ is longer than the periodicity half-length $\ell_{*} \equiv \pi \bar{R} q_{*}$ of the helical field line, an analysis similar to that used ${ }^{7}$ for showing the lowest-order axisymmetric distribution is a Maxwellian that is constant on the poloidal flux surfaces can be used to show that the appropriate solution is (127) for $|\ell| \leqslant \ell_{*}$. Conversely, for $\lambda_{e}<\ell_{*}$, finite parallel electron heat conduction ${ }^{29-31}$ limits the electron temperature equilibration to $|\ell| \leq \lambda_{e}$. A more precise description that would determine the $\ell$ dependence of $\hat{f}_{* 0}(\ell)$ in this regime, which requires a kinetic treatment similar to that developed $^{30,31}$ for calculating the parallel electron heat conduction in low-collisionality toroidal plasmas, is beyond the scope of the present paper. Next, consider the effects of the $k_{x}^{2}(\ell) \bar{D}_{\eta} \hat{f}_{*_{0}}$ term in (124). From the definition of $\bar{D}_{\eta}=\bar{\nu}_{e} \bar{\delta}_{e}^{2}$ in (73) and (66), one sees that this term is proportional to the electron collision frequency $\bar{\nu}_{e}$; it is small compared to the collision operator term on the right of (124) if $k_{x}^{2}(\ell) \bar{\delta}_{e}^{2}<1$, which yields $\ell^{2}<\ell_{\delta}^{2}$. Adding the requirement that $\ell_{*}$ be less than $\ell_{\delta}$ (so that the derivation alluded to earlier in this paragraph still applies) shows that the maximum of the two lengths $\ell_{\delta}$ and $\ell_{*}$ is the $\ell_{\max }$ defined in (123). Taking all these limits into account together with the consideration of the short periodicity length $\ell_{n^{\circ}}$ of helical field lines on loworder rational surfaces, the parallel length over which the $\hat{f}_{*_{0}}$ distribution in (127) is applicable will be written as 


$$
|\ell| \leqslant L, \quad \text { for } \hat{f}_{* 0}=f_{M}(\rho, \varepsilon, t) \text { applicability, }
$$

in which a key characteristic length has been defined:

$$
L \equiv \min \left\{\ell_{\max }, \lambda_{e}, \ell_{n^{0}}\right\}, \quad \text { equilibration length. }
$$

Note that $L$ is the minimum of three important lengths: the maximum length $\ell_{\max }$ of helical field lines diffusing radially over their entire length, the electron collision length $\lambda_{e}$, and $\ell_{n^{\circ}}$, which is the (short) half-length of helical field lines near a low-order surface where $q^{\circ}=m^{\circ} / n^{\circ}$ :

$$
\ell_{n^{\circ}}=\pi \bar{R} q_{*} n^{\circ}, \quad \text { low-order rational line length. }
$$

For low-order rational surfaces one would like to consider, for example, $m^{\circ} / n^{\circ}=2 / 1,3 / 2,3 / 1$; however, rigorously speaking, the present analysis uses an asymptotic approximation valid only for $n \gg 1$. Thus, while qualitatively $\ell$ is limited to a much shorter length in the vicinity of low-order rational surfaces, quantitatively the relevant length is only of the order of $\ell_{n^{\circ}}$ in (129).

The helically resonant radial electron heat transport induced by the radial diffusion of electron guiding centers can now be determined. The contribution in the vicinity of a $q_{*} \equiv m / n$ rational surface will be determined first. It is caused not by $f_{\text {na }}$ itself, but by the rate of change of $\hat{f}_{*_{0}}$ induced by the $\left\langle\mathcal{D}\left\{\hat{f}_{*_{0}}\right\}\right\rangle_{\theta}$ term on the right of (124):

$$
\partial \hat{f}_{*_{0}} / \partial t=\left\langle\mathcal{D}\left\{\hat{f}_{*_{0}}\right\}\right\rangle_{\theta} \simeq\left\langle\mathcal{D}\left\{f_{M}\right\}\right\rangle .
$$

Here, $\hat{f}_{*_{0}}$ is approximated by $f_{a 0}=f_{M}$ as indicated in (127) and the bounce average is approximated [correct to $\mathcal{O}\left(\epsilon^{2}\right)$ ] by the flux-surface average because there is no anisotropy ( $\mu$ dependence) in a Maxwellian distribution.

The desired helically resonant radial electron heat transport is determined from the helical Fourier component of the rate of change of the internal energy obtained from the kinetic-energy moment of $\partial f_{\text {na }} / \partial t$ :

$$
\frac{3}{2} \frac{\partial}{\partial t}\left(n_{e} T_{e}\right)_{\alpha} \equiv \int_{-\pi}^{\pi} \frac{d \alpha}{2 \pi} e^{i n \alpha} \int d^{3} v \frac{m_{e} v^{2}}{2} \frac{\partial f_{\mathrm{na}}}{\partial t} .
$$

Using the $f_{\text {na }}$ representation in (116) and $\partial \hat{f}_{* 0} / \partial t$ from (131), this rate of change in helical internal energy is

$$
\begin{aligned}
\frac{3}{2} \frac{\partial}{\partial t}\left(n_{e} T_{e}\right)_{\alpha} & =\int_{-\ell_{*}}^{\ell_{*}} \frac{d \ell}{2 \pi \bar{R} q_{*}} e^{i k_{\|}(x) \ell} \int d^{3} v \frac{m_{e} v^{2}}{2} \frac{\partial \hat{f}_{* 0}}{\partial t} \\
& \simeq \int_{-L}^{L} \frac{d \ell}{2 \pi \bar{R} q_{*}} e^{i k_{\|}(x) \ell} \int d^{3} v \frac{m_{e} v^{2}}{2}\left\langle\mathcal{D}\left\{f_{M}\right\}\right\rangle \\
& =M_{*} H_{*}(x) \frac{\partial}{\partial V}\left\langle\mathbf{Q}_{e a}^{\mathrm{pc}} \cdot \nabla V\right\rangle \equiv \frac{\partial}{\partial V}\left\langle\mathbf{Q}_{e^{*}}^{\mathrm{pc}} \cdot \nabla V\right\rangle .
\end{aligned}
$$

The following paragraphs explain the various symbols, functions, and steps involved in arriving at these results.

In going from the first to second line in (133) the limits of integration along a helical field line have been limited to $\pm L$ by a combination of the limits given in (128) and the fact that the Fokker-Planck coefficients in (83), and hence (91) and the spatial Fokker-Planck operator (95), are only applicable for $x^{2}>\bar{\delta}_{e}^{2}$ or, from (123), $|\ell| \leqslant \ell_{\max }$.

At a rational surface $x \equiv \rho-\rho_{*} \rightarrow 0,\left|k_{\|}(x) L\right| \rightarrow 0$ and one can set $e^{i k_{\|} \ell}=1$. Since, as indicated in (128), the solutions $\hat{f}_{*_{0}}(\ell)$ are reasonably constant over the length range $|\ell| \leqslant L$, for $x \rightarrow 0$ one can perform the $\ell$ integration in the second line of (133), and obtain

$$
M_{*} \equiv \int_{-L}^{L} \frac{d \ell}{2 \pi \bar{R} q_{*}}=\frac{L}{\pi \bar{R} q_{*}} .
$$

Physically, recalling the discussion in the paragraph after (117), the geometrical factor $M_{*} \sim 10 \gg 1$ enters because the distribution of electrons is Maxwellianized over $L$, which is a long distance (compared to the poloidal periodicity length $\pi \bar{R} q_{*}$ ), and as helical field lines are advected and diffused radially they carry this parallel-extended Maxwellian and equilibrated $T_{e}$ with them. Thus, as indicated by comparison of the last two lines of (133), the helically resonant paleoclassical radial electron heat transport is a multiple $M_{*}$ of the axisymmetric paleoclassical heat transport defined in (101).

The $\ell$ integral in (133) can also be performed leaving in the $e^{i k_{\|}(x) \ell}$ factor to determine the radial factor $H_{*}(x)$ :

$$
H_{*}(x) \equiv H_{m / n}(x) \simeq \frac{\sin \left[k_{\|}(x) L\right]}{k_{\|}(x) L}=\frac{\sin \left(x / x_{L}\right)}{x / x_{L}},
$$

in which the characteristic radial scale length for this autocorrelation-type function is

$$
x_{L} \equiv \frac{\bar{R} q_{*}}{L} \frac{1}{n q^{\prime}} \geqslant \frac{1}{\pi} \frac{1}{n_{\max } n q^{\prime}}=\frac{\delta x}{\pi} .
$$

The inequality follows from the definition of $L$ in (129) as the minimum of three characteristic lengths. Note that for $L=\pi \bar{R} q_{*} n_{\max }$ the function $H_{m / n}(x)$ vanishes at $|x|=\delta x$, which is the radial distance from an $\mathrm{m} / \mathrm{n}$ rational surface to the closest rational surface with $n=n_{\max }$ that was defined in (23).

In the usual paleoclassical collisionless case where $\lambda_{e}>\ell_{\max }$, when the $\hat{f}_{*_{0}}$ responses from adjacent rational surfaces are summed $\left[\Sigma_{m n} H_{m / n}(x)\right]$, they produce an overall heat transport that is nearly constant (to within about $10 \%$ ) as $x$ (or $\rho$ ) traverses many medium-order $\left(n \leqslant n_{\max } \sim 10\right)$ rational surfaces. (In summing over all possible rational surfaces the sum over $n>n_{\max }$ rational surfaces, on which the helical field lines are not diffusing and the $\hat{f}_{* 0}$ 's are not Maxwellianized over their entire length, could increase this $M_{*}=n_{\max }$ result somewhat. Also, for collisional cases where $\lambda_{e}<\ell_{\max }$ the responses overlap radially somewhat and could produce a slightly larger $M$-an effect which is however likely compensated for by a reduction in $\hat{f}_{*_{0}}$ at $|\ell| \gtrsim \lambda_{e}$ due to finite parallel electron heat conduction effects. ${ }^{30,31}$ )

Near a low-order rational surface where $n=n^{\circ}=1$ or 2 , the radial distance between the $n^{\circ}$ and $n_{\max }$ rational surfaces is larger $\left(\delta x^{\circ} \simeq 1 / n^{\circ} n_{\max } q^{\prime}\right)$. Also, because $L$ is much shorter for low-order rational field lines, the $M_{*}$ in (134) is much smaller over this larger radial range. The radial extent of this effect is largest when $q$ is at a minimum near a low-order 
rational surface since then the corresponding rational surface separation is the larger $\delta x \rightarrow \delta x_{\min }^{\circ}$ in (24) with $n \rightarrow n^{\circ}=1$ or 2.

In view of all these considerations of radial profile effects, the net (after summing over all possible rational surfaces) nonaxisymmetric paleoclassical electron heat transport varies little with radius $\rho$ from (133) with $H_{*} \rightarrow 1$ and $q_{*} \rightarrow q(\rho)$ in (134), except in the vicinity of low-order rational surfaces where it also varies little with $\rho$, but is smaller because $L$ and $M$ are smaller there. Thus, henceforth the helical factor will be defined by

$$
M \equiv \frac{L}{\pi \bar{R} q}=\frac{\min \left\{\ell_{\max }, \lambda_{e}, \ell_{n^{\circ}}\right\}}{\pi \bar{R} q(\rho)}, \text { helical multiplier. }
$$

However, since various approximations were made to obtain the net helical paleoclassical results, the factor $M$ should be regarded as a scaling result; more detailed studies are required to determine appropriate numerical, order unity "headache factors" that multiply $M$.

Paleoclassical transport and the irreversibility (entropy production) it implies are caused by the diffusion of electron guiding centers produced by the resistivity-induced poloidal magnetic-field diffusion. Thus, it does not result from collective instabilities or "fluctuations" within the plasma as microinstability-induced transport does. However, the paleoclassical model does embody small helical distortions of the otherwise axisymmetric toroidal plasma. When the plasma rotates past stationary local plasma parameter detectors these helical stuctures would cause very small oscillations on the detectors, whose amplitude will now be estimated.

First, note that $f_{\text {na }}$ just represents the distribution function along a field line [defined by $\psi=$ constant $_{1}$, $\beta=$ constant $_{2}$, as discussed near (59)] on the $t \gtrsim 1 / \nu_{e}$ time scale. To exhibit this assertion explicitly, consider the representation of $f_{\text {na }}$ in (116) using $\hat{f}_{*_{0}}$ from (127). Since the fieldline coordinate $\beta \equiv q \theta-\zeta \simeq q_{*} \theta+x q^{\prime} \theta-\zeta=-\alpha+x q^{\prime} \theta \rightarrow-\alpha$ $+k_{\|}(x) \ell / n, f_{\text {na }}$ can be written as

$$
f_{\text {na }} \simeq \sum_{n \leqslant n_{\max }} e^{i n \beta} \int_{-\ell_{*}}^{\ell_{*}} \frac{d \ell}{2 \pi \bar{R} q_{*}} \hat{f}_{* 0} \sim 2 \pi \delta(\beta) M_{*} f_{a 0} .
$$

In obtaining the last form the following sums and approximations have been used (in the limit $n_{\max } \rightarrow \infty$ ): $\sum_{n=-\infty}^{n=\infty} e^{i n \beta}$ $=2 \pi \delta(\beta)$; the $\ell$ integral is approximately $M_{*}$ and independent of $n$; and, finally, a Taylor series expansion is used for adjacent rational surface components, $\hat{f}_{* 0}(\rho) \simeq f_{a 0}\left(\rho_{*}\right)$ $+\delta x_{\tilde{n}} \partial f_{a 0} /\left.\partial \rho\right|_{\rho_{*}} \simeq f_{a 0}$ in which $\delta x_{\tilde{n}}$ is the separation between rational surfaces, i.e., a multiple of (23). The $\delta$-function factor $\delta(\beta)$ in the last form of $f_{\text {na }}$ in (138) represents the localization of this nonaxisymmetric distribution to a given value of the Clebsch field-line coordinate $\beta=q \theta-\zeta$ (i.e., to a given field line).

Neoclassical theoretical analyses ${ }^{7,8}$ usually do not determine this electron collision time-scale behavior; they implicitly assume that, since there is no "drive" for the $\hat{f}_{*_{0}}$ nonaxisymmetric components, they vanish-at least on the drift time scale. The temporal evolution of $\hat{f}_{*_{0}}$ and hence $f_{\text {na }}$ can be inferred from a next order MDKE including particle drifts in the helical (nearly toroidal) direction which tend to remove any nonaxisymmetries and hence cause $f_{\text {na }} \rightarrow 0$. Demoting the temporal evolution and paleoclassical drive $\mathcal{D}\left\{f_{M}\right\}$ to the drift time scale, the next order MDKE can be reduced using the same procedure as was used to arrive at (124) to yield $\partial \hat{f}_{*_{D}} / \partial t+i \omega_{D} \hat{f}_{*_{D}}=\left\langle\mathcal{D}\left\{f_{M}\right\}\right\rangle$ in which the effective drift frequency is $\omega_{D}(\varepsilon, \mu) \equiv i\left\langle\mathbf{v}_{D} \cdot \nabla \alpha\right\rangle_{\theta} \partial \ln f_{\text {na }} / \partial \alpha$ $\simeq-n\left\langle\mathbf{v}_{D} \cdot \boldsymbol{\nabla} \zeta\right\rangle_{\theta} \simeq k_{\theta}\left(T_{e} / e B R_{0}\right)$. Neglecting the paleoclassical drive and treating the reduced kinetic equation as an initial value problem, after Laplace transforming one obtains a Landau-type resonance $i /\left[\omega-\omega_{D}(\varepsilon, \mu)\right]$ which causes an initial $\hat{f}_{*_{D}} \simeq \hat{f}_{*_{0}}$ to be phase mixed to zero on the drift time scale $1 / \omega_{D}$ due to the drift dispersion introduced by the energy dependence of $\omega_{D}$. Thus, in the absence of a drive the nonaxisymmetric distribution function $f_{\text {na }}$ becomes negligibly small for $t \gtrsim 1 / \omega_{D}$. However, in the paleoclassical model the drive $\left\langle\mathcal{D}\left\{f_{M}\right\}\right\rangle$ causes the Maxwellianized electron distribution along a given field line to diffuse radially across the sheared magnetic field for $1 / \nu_{e} \lesssim t \lesssim 1 / \omega_{D}$; it thereby induces small helically resonant components of the electron distribution and $T_{e}$.

Helical distortions of the $T_{e}$ profile induced by nonaxisymmetric paleoclassical "drive" effects can be estimated by integrating (133) up to the drift time $1 / \omega_{D}$-after which $\hat{f}_{*_{D}}$ would be phase mixed to zero. The resultant magnitude of the helically resonant electron temperature distortion (near the $q_{*} \equiv m / n$ rational surface) is

$$
\begin{aligned}
\frac{\delta T_{e \alpha}}{T_{e}} & \sim \frac{M_{*} H_{*}(x)}{\omega_{D} T_{e}} \frac{1}{V^{\prime}} \frac{\partial^{2}}{\partial \rho^{2}}\left(V^{\prime} \bar{D}_{\eta} \frac{3}{2} T_{e}\right) \\
& \simeq \frac{\bar{\nu}_{e}}{\omega_{D}} M \bar{\delta}_{e}^{2} \frac{1}{T_{e}} \frac{\partial^{2} T_{e}}{\partial \rho^{2}} \ll 1 .
\end{aligned}
$$

Here, for simplicity, $\partial n_{e} / \partial \rho \simeq 0$ has been assumed and the small advective terms induced by $\bar{u}_{g}$ and $\bar{u}_{\psi}$ have been neglected. Since $\bar{\delta}_{e} \equiv \delta_{e} / \bar{a}$, this result is quadratically small in the ratio of the em skin depth to minor radius-because a radial diffusion process with a step size $\Delta x \sim \delta_{e}$ is involved. Also, the $H_{*}(x)$ factor in (135) localizes the $\delta T_{e \alpha}$ distortion radially to $|x| \lesssim \pi x_{L} \sim \delta x$. For typical parameters at $T_{e}$ $\sim 1 \mathrm{keV}$ of $\bar{\nu}_{e} / \omega_{D} \sim 10, M \sim 10$, and $\bar{\delta}_{e} \sim 10^{-3}$, this yields $\delta T_{e \alpha} / T_{e} \sim 10^{-4}$ over $|x| \leqq 1 / n_{\max }^{2} \sim 10^{-2}$ which is very small and radially highly localized. Thus, these $\delta T_{e \alpha}$ distortions are likely to be below experimental resolution capabilities.

\section{Near magnetic separatrix}

In a divertor configuration as one moves radially outward and approaches the magnetic separatrix, many things happen: $q$ and $q^{\prime}$ become large ( $\rightarrow \infty$ on the separatrix); the poloidal periodicity length $\pi \bar{R} q$ becomes very long and in particular longer than the collision length $\lambda_{e}$; the poloidal variation in the "straight-field-line" poloidal coordinate $\theta$ becomes highly concentrated near the $X$ point; and magneticfield lines become predominantly toroidal, especially near the $X$ point. While the preceding analyses, which implicitly 
assumed $\lambda_{e}>\pi \bar{R} q$, are no longer applicable, a related analysis can be performed in the near-separatrix region when $\pi R<\lambda_{e}<\pi \bar{R} q$

Useful coordinates near a magnetic separatrix are $\rho, \breve{\alpha}$ $\equiv \zeta / q_{*}-\theta$, and $\zeta$. (For large $q_{*}, \breve{\alpha} \simeq-\theta$.) The magnetic field here can be written as $\mathbf{B}=\boldsymbol{\nabla} \breve{\alpha} \times \nabla \psi_{t}+\nabla \zeta \times \nabla \breve{\psi}_{*}$ with $\boldsymbol{\nabla} \breve{\psi}_{*}$ $\equiv\left(1 / q-1 / q_{*}\right) \boldsymbol{\nabla} \psi_{t}$. In contrast to (106), now $\mathbf{B} \cdot \boldsymbol{\nabla} f$ $=(\mathbf{B} \cdot \boldsymbol{\nabla} \zeta)\left[\partial f / \partial \zeta-\left(1 / q-1 / q_{*}\right) \partial f / \partial \breve{\alpha}\right]$. Performing an analysis similar to that for the axisymmetric (poloidal periodicity) case above (Sec. VI B), but now based on toroidal periodicity, one obtains to lowest order $f_{s 0} \simeq f_{M}\left(\breve{\psi}_{*}, \varepsilon, t\right)$ [for $\mid(1 / q$ $\left.\left.-1 / q_{*}\right) \partial \ln f / \partial \breve{\alpha} \mid \ll 1\right]$ and a near-separatrix (subscript $s$ ) paleoclassical heat flux

$$
\left\langle\mathbf{Q}_{e s}^{\mathrm{pc}} \cdot \nabla V\right\rangle=V^{\prime}\left(\bar{u}_{g}+\bar{u}_{\dot{\psi}}\right) \frac{3}{2} n_{e} T_{e}-\frac{\partial}{\partial \rho}\left(V^{\prime} \frac{\eta_{\|}^{\mathrm{Sp}}}{\mu_{0} \bar{a}^{2}} \frac{3}{2} n_{e} T_{e}\right) .
$$

The parallel resistivity here is the Spitzer resitivity $\eta_{\|}^{\mathrm{Sp}}$ because this result is only applicable in the large $q$, nearseparatrix region where $\pi R<\lambda_{e}<\pi \bar{R} q$, which is the highcollisionality $\left(\nu_{*_{e}}>\epsilon^{-3 / 2}\right)$ Pfirsch-Schlüter regime ${ }^{7,8}$ where electron viscosity effects are negligible $\left(\mu_{e} / \nu_{e} \ll 1\right)$. This result effectively extends the axisymmetric results in (101) and (102) to the more collisional regime $\pi R<\lambda_{e}<\pi \bar{R} q$ near (but inside) a separatrix.

\section{ELECTRON ENERGY TRANSPORT}

Adding axisymmetric (neoclassical plus palaeoclassical) and nonaxisymmetric (helically resonant) paleoclassical transport effects from (101) and (133), one obtains the overall electron energy balance (in the $\mathbf{u}_{g}$ rest frame):

$$
\begin{aligned}
& \frac{3 / 2}{V^{\prime 5 / 3}} \frac{\partial}{\partial t}\left(n_{e} T_{e} V^{\prime 5 / 3}\right)+\frac{\partial}{\partial V}\left\langle\left(\mathbf{q}_{e}^{\mathrm{nc}}+\frac{5}{2} T_{e} \boldsymbol{\Gamma}_{e}^{\mathrm{nc}}\right) \cdot \nabla V\right\rangle \\
&+\frac{\partial}{\partial V}\left\langle\mathbf{Q}_{e}^{\mathrm{pc}} \cdot \nabla V\right\rangle=Q_{e} .
\end{aligned}
$$

The total paleoclassical electron heat transport with the $\bar{u}_{g}$ terms removed and neglecting the $\bar{u}_{\dot{\psi}}$ term for simplicity by assuming a near stationary poloidal magnetic field $(\dot{\psi} \simeq 0)$ can be written as

$$
\frac{\partial}{\partial V}\left\langle\mathbf{Q}_{e}^{\mathrm{pc}} \cdot \boldsymbol{\nabla} V\right\rangle=-\frac{M+1}{V^{\prime}} \frac{\partial^{2}}{\partial \rho^{2}}\left(V^{\prime} \bar{D}_{\eta} \frac{3}{2} n_{e} T_{e}\right) .
$$

The diffusive part of this electron heat transport indicates a paleoclassical electron heat diffusivity of

$$
\chi_{e}^{\mathrm{pc}} \equiv \frac{3}{2}(M+1) D_{\eta} \simeq \frac{3}{2} M \frac{\eta_{\|}^{\mathrm{nc}}}{\mu_{0}}, \text { paleoclassical } \chi_{e} .
$$

Comparing the paleoclassical electron heat diffusivity $\chi_{e}^{\mathrm{pc}}$ with the poloidal magnetic-flux diffusivity $D_{\eta}$, one sees that $T_{e}$ diffuses a factor of order $M$ faster than $\psi$ does-because of the equilibration of $T_{e}$ over the long length $L$ of the helical field lines, compared to the poloidal periodicity length $\pi \bar{R} q$. Note also that $\chi_{e}^{\mathrm{pc}}$ is applicable only for weakly transient situations where $\dot{\psi}$ satisfies (87).
There are two collisionality regimes of paleoclassical electron heat transport. They will be illustrated for a plasma whose poloidal magnetic field is in stationary equilibrium (i.e., $\dot{\psi}=0$ ). For most toroidal plasmas the collision length $\lambda_{e}$ is longer than $\ell_{\max }$; then, $M=n_{\max }=\left(\pi \bar{\delta}_{e} q^{\prime}\right)^{1 / 2} \gg 1$ yields

$$
\begin{aligned}
& \chi_{e}^{\mathrm{pc}} \simeq \frac{3}{2}\left(\frac{1}{\pi \bar{\delta}_{e}\left|q^{\prime}\right|}\right)^{1 / 2} \frac{\eta_{\|}^{\mathrm{nc}}}{\mu_{0}}, \text { collisionless pc regime }, \\
& \lambda_{e}>\ell_{\max } \equiv \pi \bar{R} q n_{\max } .
\end{aligned}
$$

This $\chi_{e}^{\mathrm{pc}}$ provides interpretations ${ }^{1-3}$ for experimentally measured $\chi_{e}$ 's in many axisymmetric toroidal plasma experiments when ${ }^{3} T_{e} \lesssim 1 \mathrm{keV} \times B(T)^{2 / 3} a(\mathrm{~m})^{1 / 2}$.

In high-density plasmas where $L$ is determined by the electron collision length $\lambda_{e}, M=\lambda_{e} / \pi \bar{R} q \gg 1$ yields

$$
\begin{aligned}
& \chi_{e}^{\mathrm{pc}} \simeq \frac{3}{2} \frac{\eta_{\|}^{\mathrm{nc}}}{\eta_{0}} \frac{v_{T e}}{\pi \bar{R} q} \delta_{e}^{2}, \text { collisional pc regime }, \\
& \pi \bar{R} q<\lambda_{e}<\pi \bar{R} q n_{\max } .
\end{aligned}
$$

This yields ${ }^{1-3}$ an Alcator-type scaling law ${ }^{32,33}$ for the plasma energy confinement time. The $\pi \bar{R} q<\lambda_{e}$ limit reflects the implicit assumption in the preceding helical paleoclassical kinetic analysis that the parallel equilibration length is longer than the poloidal periodicity length.

The collisional regime $\chi_{e}^{\mathrm{pc}}$ in (145) is similar to a formula for $\chi_{e}$ that was originally proposed by Ohkawa. ${ }^{34}$ Ohkawa's model and subsequent studies by Kadomtsev and Pogutse $^{35}$ were based on a combination of an empirical formula for $\chi_{e}$ that could reproduce the neo-Alcator confinement scaling law ${ }^{32,33}$ and a heuristic model of the collisionless effects of turbulence-induced "magnetic flutter," 21 possibly due to drift waves, within the em skin depth layer $\left(|x|<\bar{\delta}_{e}\right)$. Thus, these other models were based on physics very different from the resistivity-induced magnetic-fieldline diffusion outside the em skin depth layer $\left(|x|>\bar{\delta}_{e}\right)$ and collisional regime $\left(\lambda_{e}\right.$-limited $)$ paleoclassical model presented here.

In the near-separatrix region where $q$ and $q^{\prime}$ become very large, one can have $\lambda_{e} \lesssim \pi \bar{R} q$. In this region, combining the results in (140) and (142), the paleoclassical electron heat diffusivity becomes

$$
\begin{aligned}
& \chi_{\mathrm{es}}^{\mathrm{pc}} \simeq \frac{3}{2} \frac{\eta_{\|}^{\mathrm{Sp}}}{\mu_{0}}\left(1+\frac{\eta_{\|}^{\mathrm{nc}}}{\eta_{\|}^{\mathrm{Sp}}} \frac{\lambda_{e}}{\pi \bar{R} q}\right), \text { near separatrix }, \\
& \pi R<\lambda_{e}<\pi \bar{R} q \max \left\{1, n_{\max }\right\} .
\end{aligned}
$$

For $\lambda_{e} / \pi \bar{R} q>\left(\eta_{\|}^{\mathrm{Sp}} / \eta_{\|}^{\mathrm{nc}}\right) \sim 1$ this yields the collisional (Alcator scaling) $\chi_{e}^{\mathrm{pc}}$ in (145). In the opposite limit, which applies where $q$ gets very large near the separatrix, one obtains a smaller $\chi_{\mathrm{es}}^{\mathrm{pc}} \simeq(3 / 2)\left(\eta_{\|}^{\mathrm{Sp}} / \mu_{0}\right) \simeq Z_{\mathrm{eff}}\left[100 / T_{e}(\mathrm{eV})\right]^{3 / 2} \mathrm{~m}^{2} / \mathrm{s}$. Encouraging qualitative comparisons of the paleoclassical model with experimental $T_{e}$ profile data in the edge region just inside the separatrix are discussed briefly in Refs. 2 and 3. 
The paleoclassical model derived in this paper applies to all types of axisymmetric toroidal plasmas-tokamaks, spherical tokamaks (STs), spheromaks, and reversed field pinches (RFPs)—in regions where $\epsilon^{2}, B_{p}^{2} / B_{t}^{2} \ll 1$. Encouraging preliminary, qualitative comparisons of $\chi_{e}^{\mathrm{pc}}$ predictions with experimental $\chi_{e}$ 's inferred from axisymmetric toroidal plasma experiments other than tokamaks are discussed in Refs. 2 and 3.

As indicated by (129), (137), and (143), the predicted $\chi_{e}^{\mathrm{pc}}$ is much smaller in the vicinity of low-order rational surfaces with $q^{\circ}=m^{\circ} / n^{\circ}: \chi_{e}^{\mathrm{pc}} \sim(3 / 2)\left(n^{\circ}+1\right) \eta_{\|}^{\mathrm{nc}} / \mu_{0}$. Over what radial width does this "transport barrier" exist? From the discussion in the paragraph after (136), the width of the transport barrier is

$$
2 \delta x^{\circ} \simeq \frac{2 / n^{\circ}}{n_{\max }\left|q^{\prime}\right|}=\frac{2}{n^{\circ}}\left(\frac{\pi \bar{\delta}_{e}}{\left|q^{\prime}\right|}\right)^{1 / 2}, \text { barrier width. }
$$

Or, if $q$ is very near a minimum at the rational surface,

$$
2 \delta x_{\min }^{\circ} \simeq 2\left(\frac{2}{n^{\circ}}\right)^{2 / 3}\left(\frac{\pi \bar{\delta}_{e}}{q^{\prime \prime}}\right)^{1 / 3}, \text { width at } q_{\min } .
$$

These widths compare favorably ${ }^{2,3}$ with those obtained experimentally in a number of toroidal plasmas with electron internal transport barriers.

The paleoclassical electron heat transport in (142) is not in a normal (diffusive) Fourier heat flux law form (i.e., $\boldsymbol{\nabla} \cdot \mathbf{q}_{e}$ with $\mathbf{q}_{e}=-\kappa_{e} \boldsymbol{\nabla} T_{e} \equiv-n_{e} \chi_{e} \boldsymbol{\nabla} T_{e}$ ). Rather, neglecting the spatial variation of $M$ (so it can be taken inside the derivatives with respect to $\rho$ ), the implied paleoclassical heat flux can be written approximately in terms of (normal, Fourier) diffusive and (abnormal) "heat pinch" fluxes:

$$
\begin{aligned}
& \left\langle\mathbf{Q}_{e}^{\mathrm{pc}} \cdot \nabla V\right\rangle \approx-V^{\prime} n_{e} \bar{X}_{e}^{\mathrm{pc}} \frac{\partial T_{e}}{\partial \rho}-\left\langle\mathbf{q}_{e}^{\mathrm{pi}} \cdot \nabla V\right\rangle, \\
& \left\langle\mathbf{q}_{e}^{\mathrm{pi}} \cdot \nabla V\right\rangle \equiv T_{e} \frac{\partial}{\partial \rho}\left(V^{\prime} n_{e} \bar{X}_{e}^{\mathrm{pc}}\right), \text { heat pinch, }
\end{aligned}
$$

in which $\bar{\chi}_{e}^{\mathrm{pc}} \equiv \chi_{e}^{\mathrm{pc}} / \bar{a}^{2}$. This electron heat pinch heat flux ${ }^{36}$ is usually positive (inward) and increases with $\rho$, in qualitative agreement with experimental inferences. ${ }^{3}$ Alternatively, in qualitative agreement with some tokamak experimental data, ${ }^{37}$ the approximate $\left\langle\mathbf{Q}_{e}^{\mathrm{pc}} \cdot \boldsymbol{\nabla} V\right\rangle$ can be written as a heat flux proportional to the degree to which $\left|\nabla \ln T_{e}\right| \equiv 1 / L_{T_{e}}$ exceeds a critical magnitude:

$$
\left.\frac{1}{L_{T_{e}}}\right|_{c} \simeq \frac{\partial}{\partial r} \ln \left(V^{\prime} n_{e} \bar{D}_{\eta}\right), \text { critical gradient of } \ln T_{e} .
$$

There has been a long-standing mystery ${ }^{38-40}$ as to why transiently measured electron heat diffusivities usually exceed those determined from equilibrium "power balance" measurements. A straightforward perturbation $\left(T_{e} \rightarrow T_{e 0}\right.$ $\left.+\widetilde{T}_{e}\right)$ of the paleoclassical electron heat transport in (142) would yield a "heat pulse" electron heat diffusivity that is negative since (neglecting $\nu_{*_{e}}$ effects) $T_{e} D_{\eta} \propto T_{e}^{-1 / 2}$. However, transient electron heat transport induced by radially lo- calized perturbations can be considered with the present paleoclassical model only when $\dot{\psi}$ satisfies conditions (87). Many transiently measured $\chi_{e}$ 's use sawteeth-generated heat pulses or localized electron heating [e.g., due to electron cyclotron resonance heating (ECRH)] to induce radially localized transients in $T_{e}$. These effects change the local $T_{e}$ and hence the resistivity $\eta_{\|}^{\text {nc }}$; thus, from the definition of $\dot{\psi}$ in (80), they are very likely to violate conditions (87), particularly the first one. Further, since for $\dot{\psi} \neq 0$ these transient experiments change the toroidal inductive electric field in the plasma, they also change the $\mathbf{E} \cdot \mathbf{J}$ joule heating embodied in the $Q_{e}$ collisional heating term ${ }^{7,8}$ in the electron energy balance equation (141). A T-10 experiment ${ }^{41}$ that surely violated conditions (87) observed a large inward "ballistic jump in the total heat flux just after on-axis ECRH switching on." A full transient transport paleoclassical analysis that selfconsistently includes all possible effects due to rational fluxsurface motion, local helical flux distortions, inductive electric-field perturbation effects, and changes in the joule heating is beyond the scope of the present paper. However, the profound question that results from this discussion is can one ever measure the local electron heat diffusivity via electron heating or localized transient responses? The answer seems to be only if $T_{e}$ can be perturbed in a way that causes small, relatively homogeneous changes in the poloidal magnetic flux $\psi(\rho, t)$ that satisfy conditions (87).

\section{SUMMARY AND DISCUSSION}

As emphasized in the first part of Sec. VI, the key hypothesis of the paleoclassical model is that the electron guiding centers are advected and diffused radially with the same Fokker-Planck coefficients as the poloidal magnetic flux is for current-carrying resistive toroidal plasmas (i.e., $\eta_{\|}\langle\mathbf{J} \cdot \mathbf{B}\rangle$ $\neq 0$ ), namely, $(83) \rightarrow(91)$. This effect is taken into account by adding a spatial Fokker-Planck diffusion operator to the the usual drift-kinetic equation, as indicated in (92). The main results obtained from this fundamental hypothesis for driven-dissipative magnetic confinement systems (e.g., tokamaks) are in (142)-(151). They are supplemented by definitions of the: multiplier $M$ in (137), equilibration length $L$ in (129), magnetic-field diffusivity $D_{\eta}$ in (66), and neoclassical parallel resistivity $\eta_{\|}^{\text {nc }}$ in (46)-(53). For transients these formulas are applicable only for a slowly changing (in space and time) poloidal flux $\psi(\rho, t)$ that satisfies conditions (87). Since the results were obtained using a large $n$ asymptotic analysis and the characteristic lengths in $L$ were only approximately determined, the formula for $M$, and hence all $M$-dependent results herein, should be interpreted as scaling results; more detailed studies beyond the scope of this paper could introduce numerical coefficients of order unity in $L$, $M$, and $\chi_{e}^{\mathrm{pc}}$.

The results in (141)-(145) reduce to those obtained previously ${ }^{1}$ with a slab model in the limit of a large aspect ratio tokamak when the poloidal magnetic field is stationary. The initial paper ${ }^{1}$ elucidated how the resultant, simplest paleoclassical model provides interpretations for many aspects of the experimentally observed electron heat diffusivity: magnitude, multiple of magnetic-field diffusivity $D_{\eta}$, radial 
profile, neoclassical collisionality regime, Alcator scaling law in high-density tokamak plasmas, and reduced $\chi_{e}$ 's near low-order rational surfaces and just inside a magnetic separatrix. Companion papers ${ }^{2,3}$ discuss promising comparisons of the more general formulas developed in this paper with the $\chi_{e}$ 's inferred from tokamaks, spherical tokamaks (STs), and quiescent reversed field pinch (RFP) plasmas. And for tokamak plasmas, the paleoclassical model provides interpretations ${ }^{2,3}$ for radial widths and depths of electron transport barriers near low-order rational $q$ minima, $\chi_{e}$ in near-separatrix regions, and heat pinch or minimum temperature gradient forms of the electron heat flux.

The main contributions of this paper have been to (1) develop the paleoclassical transport model for a full axisymmetric toroidal magnetic-field geometry (assuming $\left.\epsilon^{2}, B_{p}^{2} / B_{t}^{2} \ll 1\right)$, (2) present evolution equations for the poloidal magnetic flux $\psi$, the toroidal flux $\psi_{t}$ and the local helical flux $\psi_{*}$, and their interelationships, (3) develop an appropriate parallel Ohm's law for the paleoclassical model that includes neoclassical and inertial effects, (4) clarify the local diffusion properties of the poloidal flux $\psi$ and, near rational flux surfaces, the helical flux $\psi_{*}$, (5) determine the limits on $\dot{\psi}$ [in (87)] for application of the paleoclassical model to transient electron heat transport studies, (6) explain how electron gyromotion radially across poloidal flux surfaces combined with radial diffusion of poloidal flux (field lines) induced by resistivity in driven-dissipative poloidal magnetic-field systems (e.g., tokamaks) leads to radial diffusion of the usual $p_{\zeta} \propto \psi$ constant of the motion and hence of the electron guiding centers, (7) develop a low-collisionality kinetic analysis of paleoclassical processes based on adding a spatial Fokker-Planck operator to the electron drift-kinetic equation to take account of the electron guiding center diffusion induced by the radial diffusion of the poloidal magnetic flux in current-carrying toroidal systems, (8) explore the parallel extent of the Maxwellianization of the electron distribution and $T_{e}$ equilibration along poloidal and helical field lines, (9) determine the axisymmetric and helically resonant radial electron heat transport induced by the diffusing poloidal flux, (10) clarify the ballooning representation of the nonaxisymmetric (helically resonant) distribution function which yields the multiplier $M$ that reflects the long distance along field lines over which $T_{e}$ is equilibrated, (11) estimate the radial variation of $\chi_{e}^{\mathrm{pc}}$ and width of "internal transport barriers" where $\chi_{e}^{\mathrm{pc}}$ is significantly reduced in the vicinity of low-order rational surfaces, and (12) illustrate how heat pinch or minimum electron temperature gradient forms emerge naturally from the paleoclassical transport model.

Because paleoclassical electron heat transport is based on the primitive Coulomb collision processes of parallel electron heat conduction and electrical resistivity leading to magnetic-field diffusion, it should be considered an "irreducible, ubiquitous" plasma transport process, just as classical and neoclassical transport ${ }^{7,8}$ are. Since $D_{\eta} \propto \eta \propto 1 / T_{e}^{3 / 2}$, "collisionless" paleoclassical electron heat diffusion decreases with increasing electron temperature; for $n_{\max } \leqslant 10$ and $\eta_{\|}^{\mathrm{nc}} / \eta_{0} \lesssim 2, \chi_{e}^{\mathrm{pc}} \sim a^{1 / 2} / T_{e}^{3 / 2}$ becomes less than $1 \mathrm{~m}^{2} / \mathrm{s}$ for $T_{e}$ $\gtrsim 2 \mathrm{keV}$. In contrast, microturbulence-induced transport due to ion temperature gradient, trapped electron, or electron temperature gradient microinstabilities, which typically result in a gyro-Bohm scaling of $\chi_{e}^{\mu \text { turb }} \sim(\varrho / a)\left(T_{e} / e B\right)$ $\sim T_{e}^{3 / 2} / a B^{2}$, increase with $T_{e}$. Equating the two scalings, one infers paleoclassical electron heat transport may be dominant $^{3}$ for $T_{e} \leqq 1 \mathrm{keV} \times B(T)^{2 / 3} a(\mathrm{~m})^{1 / 2}$. Microturbulence would apparently not affect paleoclassical processes since it usually does not significantly affect the parallel Ohm's $\operatorname{law}^{42,43}$ and the parallel correlation length for magnetic microturbulence usually exceeds the relevant paleoclassical parallel length $L$.

\section{ACKNOWLEDGMENTS}

The author is grateful to many of his colleagues for their comments and constructive suggestions in response to seminars and talks he has given on this research at many universities, laboratories, and conferences over the past year. He is particularly grateful to K.C. Shaing for suggesting the stochasic differential equation (Fokker-Planck) approach, to C.C. Hegna for clarifications of geometrical effects, to D.G. Whyte and T.H. Osborne for stimulating consideration of the near separatrix region, and to the U.S. Department of Energy for support of his research over the past three decades.

${ }^{1}$ J. D. Callen, Phys. Rev. Lett. 94, 055002 (2005).

${ }^{2}$ J. D. Callen, Fusion Energy 2004 (IAEA, Vienna, 2004), paper TH/1-1.

${ }^{3}$ J. D. Callen, "Nucl. Fusion 45, 1120 (2005).

${ }^{4}$ H. Grad and J. T. Hogan, Phys. Rev. Lett. 24, 1337 (1970).

${ }^{5}$ Y. Pao, Phys. Fluids 19, 1177 (1976).

${ }^{6}$ Y. Pao, Phys. Fluids 21, 1120 (1978).

${ }^{7}$ F. L. Hinton and R. D. Hazeltine, Rev. Mod. Phys. 48, 239 (1976).

${ }^{8}$ S. P. Hirshman and D. J. Sigmar, Nucl. Fusion 21, 1079 (1981).

${ }^{9}$ S. P. Hirshman and S. C. Jardin, Phys. Fluids 22, 731 (1979).

${ }^{10}$ P. I. Strand and W. A. Houlberg, Phys. Plasmas 8, 2782 (2001).

${ }^{11}$ J. D. Callen and R. A. Dory, Phys. Fluids 15, 1523 (1972).

${ }^{12}$ J. P. Freidberg, Ideal Magnetohydodynamics (Plenum, New York, 1987).

${ }^{13}$ J. P. Wang and J. D. Callen, Phys. Fluids B 5, 3207 (1993).

${ }^{14}$ A. L. Garcia-Perciante, J. D. Callen, K. C. Shaing, and C. C. Hegna, Phys. Plasmas 12, 052516 (2005).

${ }^{15}$ C. T. Hsu, K. C. Shaing, R. P. Gormley, and D. J. Sigmar, Phys. Fluids B 4, 4023 (1992).

${ }^{16}$ L. Spitzer, Physics of Fully Ionized Gases (Interscience/Wiley, New York, 1962).

${ }^{17}$ S. P. Hirshman, R. J. Hawryluk, and B. Birge, Nucl. Fusion 17, 611 (1977).

${ }^{18}$ C. C. Hegna, Phys. Plasmas 6, 3980 (1999).

${ }^{19}$ S. Chandrasekhar, Rev. Mod. Phys. 15, 1 (1943).

${ }^{20}$ N. G. Van Kampen, Stochastic Processes in Physics and Chemistry (North-Holland, Amsterdam, 1981).

${ }^{21}$ J. D. Callen, Phys. Rev. Lett. 39, 1540 (1977).

${ }^{22}$ R. D. Hazeltine and J. D. Meiss, Plasma Confinement (Dover, New York, 2003).

${ }^{23}$ M. N. Rosenbluth, W. M. MacDonald, and D. L. Judd, Phys. Rev. 107, 1 (1957).

${ }^{24}$ J. W. Connor, R. J. Hastie, and J. B. Taylor, Phys. Rev. Lett. 40, 396 (1978).

${ }^{25}$ Y. C. Lee and J. W. Van Dam, Proceedings of the Finite Beta Workshop (Societa Italiana di Fisica, Bologna, 1979), p. 93. See National Technical Information Service Document No. CONF-7709167. Copies may be ordered from the National Technical Information Service, Springfield, VA 22161.

${ }^{26} \mathrm{P}$. M. Morse and H. Feshbach, Methods of Theoretical Physics (McGrawHill, New York, 1953).

${ }^{27}$ J. W. Connor and R. J. Hastie, Phys. Rev. Lett. 92, 075001 (2004).

${ }^{28}$ J. W. Connor and R. J. Hastie, Phys. Plasmas 11, 1520 (2004). 
${ }^{29}$ S. I. Braginskii, Reviews of Plasma Physics (Consultants Bureau, New York, 1965), Vol. I, p. 205.

${ }^{30}$ E. D. Held, J. D. Callen, C. C. Hegna, and C. R. Sovinec, Phys. Plasmas 8, 1171 (2001).

${ }^{31}$ E. D. Held, J. D. Callen, and C. C. Hegna, Phys. Plasmas 10, 3933 (2003).

${ }^{32}$ M. Greenwald, D. Gwinn, S. Milora et al., Phys. Rev. Lett. 53, 352 (1985).

${ }^{33}$ B. B. Kadomtsev, Tokamak Plasma: A Complex Physical System (IOP, Bristol, 1993), p. 103.

${ }^{34}$ T. H. Ohkawa, Phys. Lett. A 67, 35 (1978).

${ }^{35}$ B. B. Kadomtsev and O. P. Pogutse, Plasma Physics and Controlled Nuclear Fusion Research 1978 (IAEA, Vienna, 1979), Vol. I, p. 649.
${ }^{36}$ J. D. Callen, J. P. Christiansen, J. G. Cordey, P. R. Thomas, and K. Thomsen, Nucl. Fusion 27, 1857 (1987).

${ }^{37}$ G. T. Hoang, W. Horton, C. Bourdelle, B. Hu, X. Garbet, and M. Ottaviani, Phys. Plasmas 10, 405 (2003).

${ }^{38}$ J. D. Callen and G. L. Jahns, Phys. Rev. Lett. 38, 971 (1977).

${ }^{39}$ E. D. Fredrickson, J. D. Callen, K. McGuire et al., Nucl. Fusion 26, 849 (1986).

${ }^{40}$ N. J. Lopes Cardozo, Plasma Phys. Controlled Fusion 37, 799 (1995).

${ }^{41}$ V. F. Andreev, Y. N. Dnestrovskij, M. V. Ossipenko, K. A. Razumova, and A. V. Suskov, Plasma Phys. Controlled Fusion 46, 319 (2004).

${ }^{42}$ K. C. Shaing, Phys. Fluids 31, 8 (1988).

${ }^{43}$ F. L. Hinton, R. E. Waltz, and J. Candy, Phys. Plasmas 11, 2433 (2004). 